\title{
CHALLENGES AND STATUS ON DESIGN AND COMPUTATION FOR EMERGING ADDITIVE MANUFACTURING TECHNOLOGIES
}

\author{
Yuen-Shan Leung ${ }^{1}$, Tsz-Ho Kwok ${ }^{2}$, Xiangjia Li $^{1}$, Yang Yang ${ }^{1}$, Charlie C.L. Wang ${ }^{3}$, Yong Chen*1 \\ ${ }^{\prime}$ Epstein Department of Industrial and Systems Engineering, University of Southern California, USA \\ ${ }^{2}$ Department of Mechanical, Industrial and Aerospace Engineering, Concordia University, Canada \\ ${ }^{3}$ Department of Machanical and Automation Engineering, The Chinese University of Hong Kong, Hong Kong, China
}

\begin{abstract}
The revolution of additive manufacturing (AM) has led to many opportunities in fabricating complex and novel products. The increase of the printable materials and the emergence of the various fabricating processes continuously expand the capability of manufacturing. Our products are no longer limited to be single material, single scale or single function. In fact, a paradigm shift is taking place in the industries from geometry-centered usage to support functional demands, and hence it is expected to resolve wide range of complex and difficult problems. Although AM provides us higher design degree of freedom beyond the geometry to fabricate new objects with tailored properties and functions, there are only very few approaches for computational design in this new domain enabled by AM. The objectives of this study are to provide an overview on the current computer-aided design methodologies that are applied to multi-material, multiscale, multi-form and multi-functional AM technologies. We summarize the difficulties encountered in the design approaches and emphasize the need for the future development. The study also introduces the related manufacturing processes, lists their present applications, and discusses their potential future trends.
\end{abstract}

\section{INTRODUCTION}

Additive manufacturing (AM), a.k.a. 3D printing, has been around for decades. The technology has come on leaps and bounds in the past 10 years, provided an alternative to traditional manufacturing methods to produce parts. The capabilities of creating complex internal geometries give engineers more options to innovate without being limited by traditional manufacturing methods. Recently, the technology has evolved from geometry-focused fabrication to produce functional parts of varying material composition and scales. The increased interdisciplinary interactions and the urgent need for practical applications have put forward new demands. Nowadays, fabrication of a multifunctional structure is made practical by the next generation of additive manufacturing technologies, enable advances in products across a wide range of applications.

Develop appropriate tools to design a part with functional consequences is urgently needed. Existing CAD software is developed for traditional manufacturing, where geometry is their major concern. Although some of them allow users to assign material properties on models, the operations cannot guarantee the functional capability, especially when the functions can hardly be realized or optimized by manual design activities. Therefore, most of the current research still use CAD software coupling with finite element analysis (FEA) and trial-and-error approach to design functional properties. At the same time, a growing number of researchers are developing computational methods to help automate the design with functionality.

Many researchers have extensively studied in changing geometry shape to alter an object's property. Commercial software like OptiStruct [1] have been developed to help designers to create conceptual designs with target structural properties. On top of this, researchers try to alter material compositions to create ever complicated properties. Varying material compositions within layers of print can now be achieved through 
controlling process parameters. This additional design degree of freedom offers new and significant solutions to many industry sectors, such as aerospace [2, 3], defense [4], biomedical [5, 6], wearable devices [7] and tissue engineering [8].

In additive manufacturing, the internal structure can be optimized in multiple scales to satisfy functional requirements. Through optimizing structural parameters, the multi-scale structures can offer multifunctional properties. For example, the wettability of an object is changed from hydrophobic to superhydrophobic when the surfaces are embedded with micro-pillars [9]. In addition, new type of material with microstructure based on variational unit cells, the properties of which can be programed upon requirement, have emerged as an alternative to fulfil the complex functional demand. Coupling with recent multiscale additive manufacturing techniques, programming the world of material is becoming possible.

As the multi-material and multi-scale printing capabilities continue to grow, people move on consider other properties, such as thermal, optical, acoustic or electrical, combining with the desired geometry shape, to create multi-functional objects. The additional functionalities add even more challenge to the designers. Not only facing interdisciplinary problems, but also the unique criteria and characters in different fabrication processes. At this moment, systematic design methodology is inadequate, leading to inefficiencies in the design to manufacturing process.

This paper reviews recent research on fabricating functional objects by AM technologies with an emphasis on their design methodology. We classify them into four categories, according to the design requirements and constraints. While some research addresses multiple constraints (e.g. Bio-compatibility and mechanical constraints), we will focus on one constrain that they are mainly designed for, such as

1) Multi-Material: Design for enhancing mechanical properties or material properties (e.g. color) by depositing multiple dissimilar materials within one single build. (Section 3.1)

2) Multi-Scale: Design for achieving functional requirements by printing materials in multiple scales. (Section 3.2)

3) Multi-Form: Design for programmed shape changing after fabrication. (Section 3.3)

4) Multi-Functional: Design for objects with non-structural requirements. The objects can have structural requirements as well but mainly focus on the non-structural one. (Section 3.4)

The purpose of this paper is to give an overview of the current status of functional design in additive manufacturing. We summarize the existing challenges faced by designers, suggest best practices for the design community, and mention future directions.

\section{RECENT DEVELOPMENT ON AM TECHNOLOGIES}

Additive manufacturing technologies have been extensively developed in the past few years, with specific techniques being applied to various 3D geometric objects, in the fashion of depositing different materials layer upon layer [10]. Compared with traditional manufacturing methods, such as CNC machining and casting, injection molding, plastic forming and joining, additive manufacturing is more flexible to build complex 3D object in a short period of time [11, 12]. Due to its unique advantages, AM technology has been widely applied in the fields of manufacturing, medical, industry and sociocultural sectors [13]. This section provides an overview of recent developments and status on future AM technologies. We will expound four promising directions for the future development of AM technologies: multi-material printing, multi-scale printing, multi-functional printing and 4D printing (i.e., 3D printing time-variant structures). Discussions are mainly based on the process development, the fabrication requirements and the promising applications of additive manufacturing technologies. 


\subsection{MULTI-MATERIAL AM PROCESSES}

A vast array of multi-materials structures and systems have functional and innovative applications in various areas. For example, multi-material composite structures with shape-changing function, bioinspired multi-material composites with promising mechanical performance, multi-material scaffold for tissue regeneration, and multi-material fiber with attractive optical applications [14]. Overall, the multi-material structure found in nature gives us many inspirations and also design principles for the construction of multifunctional system. Fabrication of novel multi-materials, for functional integration of two or more biological materials, has become increasingly significant. However, traditional manufacturing methods can only provide limited possibility to fabricate 3D structures with complex spatial multi-material distributions. Besides, the functional performance of fabricated structures is also limited by the fabrication resolution of traditional manufacturing process. Due to the processing capability, additive manufacturing technology provides a promising solution to fabricate multi-material structures with high resolution and complex geometric shape. Meanwhile, attractive complex natural structures with multi-material also drive the demand for developing more advanced multi-material additive manufacturing processes.

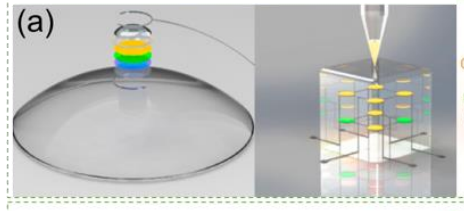

(b)

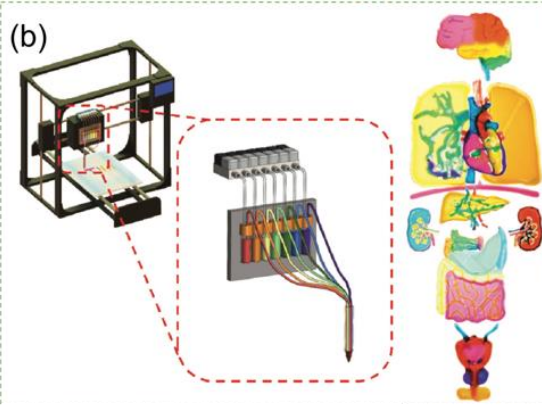

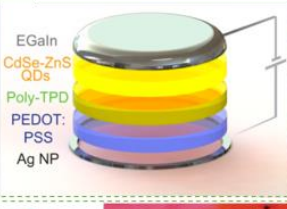

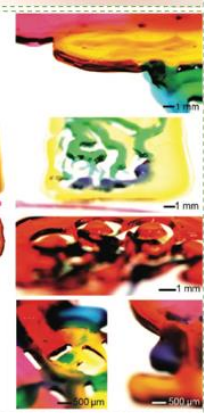

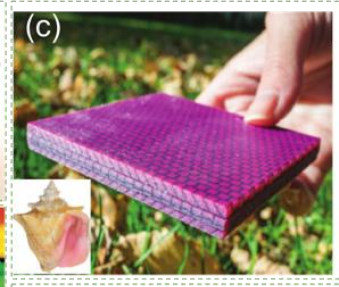

(d)

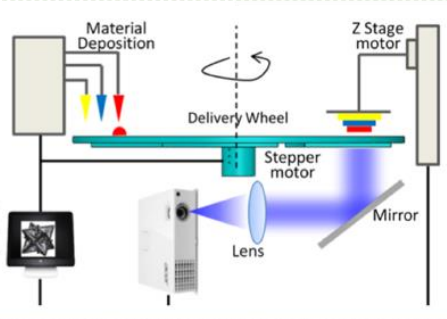

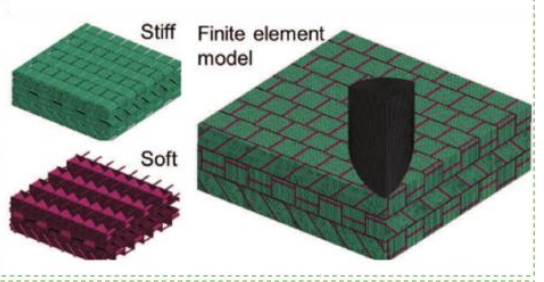

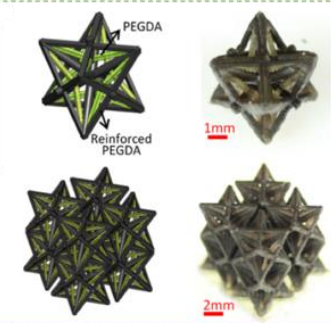

Figure 1 (a) Direct 3D printing of QD-LEDs on a substrate [15]. (b) Design of a digitally tunable continuous multi-material extrusion bio-printer and the side view of selected organ-like constructs [16]. (c) 3D printed nacre-inspired sample and Quarter geometry of the Nacre-like design in simulation [4]. (d) Three-dimensional fabricated negative thermal expansion lattice structures using multi-material projection micro-sterelithography system [17].

\subsubsection{Stereolithography (SLA) based Multi-Material 3D Printing}

In most of extrusion-based 3D printing processes, thermoplastic is utilized and the state of material alters from liquid to solid during the extrusion process as a result of changing thermal condition $[18,15$, 19]. Different from thermoplastic, photo-curable polymer is solidified with sufficient light exposure. Stereolithography is such a 3D printing process to build 3D object using photo-curable polymer. For instance, based on material jetting technologies, 3D multi-material inkjet printing is developed to fabricate complicated structures using photopolymer and wax. Micro-scale droplets of photo curable ink are deposited layer by layer in inkjet printing process, and droplets can be turned to solid after receiving enough energy from light exposure [4, 20,21]. Due to the high resolution of mature jetting technology, the surface quality of objects printed by polyjet/inkjet printing is much better than the one built by FDM processes. In addition, high-resolution multi-material 3D printing brings a new future to the optical application. Biomimic imaging optical eye and display window, which is not easy to be fabricated using traditional methods, were fabricated by inkjet printing using transparent acrylic polymer [22]. SLA-based multi-material 3D printing can also achieve superior mechanical performance that cannot be provided by single material printing. Biological structures found in nature give us inspirations that soft and hard materials can be 
allocated in a distributed pattern to achieve certain mechanical performance. Multi-material 3D printing processes enable the improvement of structural properties with special design of material distribution. Gu et al. [4] used an Objet 500 3D printer to replicate the innate toughness of the nacre and a conch shell. In the nacre-like designs, two base materials, which are vastly different in properties, were assembled in a ply with an architecture similar to nacre. These plies were then stacked with orientation angles of 0 and 90 degrees to generate a laminate structure by multi-material 3D printing (Figure 1(c)). Similarly, prototypes of mixing stiff plate and soft matrix of bone-like plate, mollusk shells shaped structures, and ganoid fish scales were fabricated by the inkjet printing process, and the printed part demonstrates promising mechanical performance [21].

Instead of curing droplets, one layer of photo curable polymer can be solidified by the exposure of the 2D patterned light beam in mask image projection based stereolithography (MIP-SLA) [23]. Compared with other AM methods, the building speed of MIP-SLA process is relatively fast by taking advantages of high resolution 2D patterned light beam. Therefore, numerous studies are conducted to develop MIP-SLA based multi-material processes $[17,24,25,26]$. With special design of the layouts of different material, the structure can be controlled to bend or twist under different environmental situations. Using multi-material 3D printing techniques, such as inkjet or SLA processes, shape-changing anisotropy can be achieved by combining a responsive shape-changing material with a passive matrix material [24]. Similarly, the thermal performance of 3D printed parts can be manipulated by material allocation. As shown in Figure 1(d), negative thermal explosion micro light weight multi-material lattices structures were printed by maskimage projection-based SLA printing process, with different mixture ratio of photo-curable polymer and copper nanoparticles solutions [17]. According to the fabrication requirements, new material is easily to be added in the SLA based system by increasing the number of material reservoirs. Current multi-material MIP-SLA process is rather slow, since the residual material removal is mandatory prior to the placement of new material. Due to insufficient removal force as well as adhesive forces between resin molecules and a cured part, incoming new material tends to mix with the residual, and generate blur mixtures attached to the cured part. Such blur mixtures existing in the interface area of different materials usually affects the performance of fabricated objects, and is still a challenge for researchers to overcome in future.

\subsubsection{Other Multi-Material 3D Printing Methods}

Unlike traditional machining process, i.e. subtractive manufacturing, raw material is gradually deposited to build an object from scratch in AM process. Based on the deposition principle, multi-material 3D objects can be constructed by using AM processes with multiple material supplies. For example, multimaterial fused disposition modeling (FDM) process is developed that different materials are extruded from different extruders [18]. Using material extrusion methods, a broad variety of material such as thermoplastics, ceramic slurries and metal pastes can be printed using FDM process. Moreover, structures with a lot of overhangs can be easily printed using a multi-material FDM machine with dissolvable supporting material. However, this inexpensive multi-material AM solution is limited with poor fabrication resolution due to the dimension of extruder [27, 28, 29]. To get better fabrication resolution, another kind of $3 \mathrm{D}$ printing method is developed to fabricate multi-material structures, using only a special designed print head $[15,16,19]$. One kind of print head, consisted of a bundle of capillaries in connection with multiple printing-ink reservoirs, was designed to continuously actuate digital material under controllable pneumatic pressure, as shown in Figure 1(b). Cells, culture matrix, and growth factor were continuously extruded together into specially designed organ structures, and the bio-material kept bioactivities during the extrusion process. 3D structures with multi-material show multiple functional performances because each type of material has its own physical property. Multi-material 3D printing process enables the fabrication of active functional structures with multiple materials to achieve interesting applications. Traditionally, single material, such as plastic polymer, conductors, and biological material, can be easily 
printed using extrusion-based process. Five different types of materials including semiconducting nanoparticles, elastomer, organic polymer, solid and liquid metal, and UV curable polymer are integrated together by extrusion-based process to build a functional quantum dot light-emitting diode (Figure 1(a)) [15]. Extrusion-based multi-materials 3D printing process offers the capability of highly flexible fabrication, and the ability of directly depositing multiple functional material enables us to build semiconducting electronic device with complex geometric shape. In addition to the aforementioned processes, which are mainly developed to fabricate liquid based material, researchers have also developed powder based multimaterial printing processes. For example, binder jetting printing method is developed to fabricate powder based multi-material structures, such as polymer powder, ceramic powder and metal powder [30].
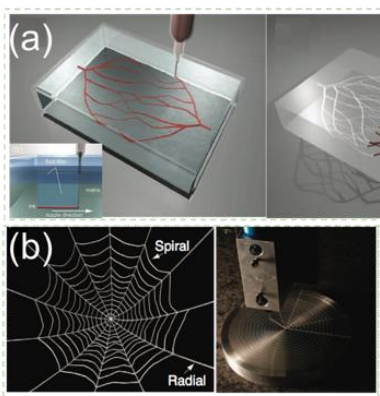

(d)

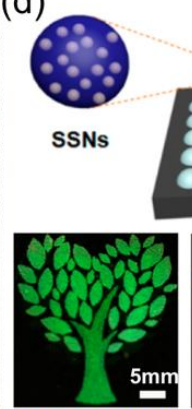

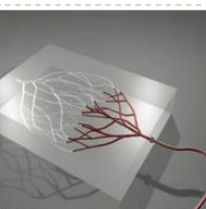
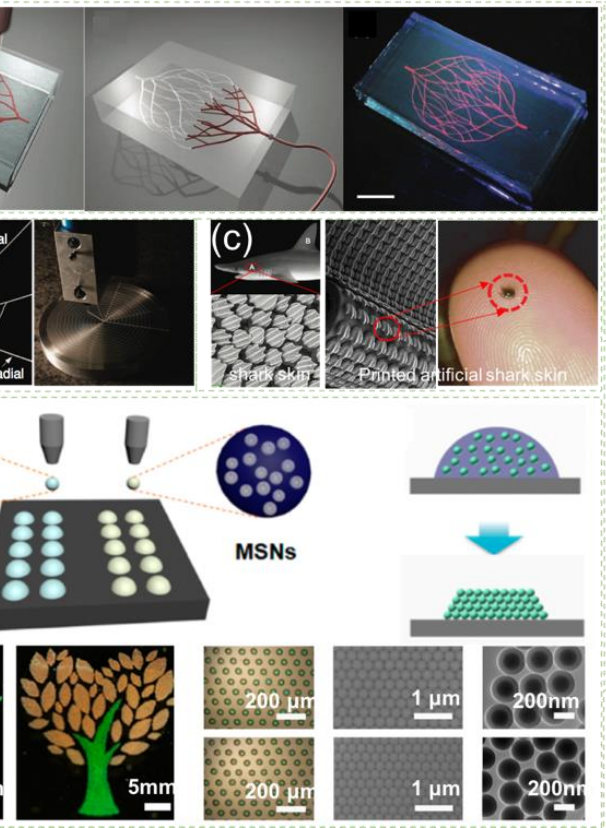

(e)
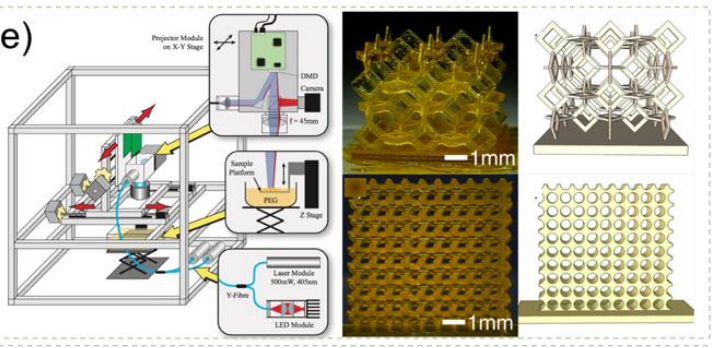

(f)

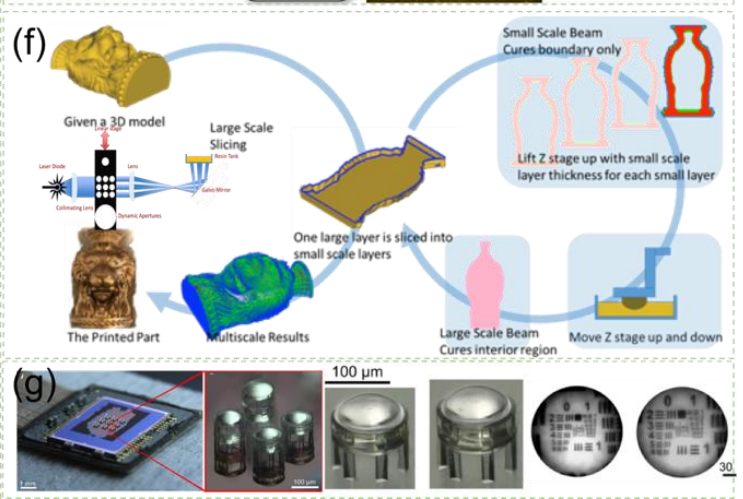

Figure 2 (a) Omnidirectional printing of 3D microvascular networks within a hydrogel reservoir [31]. (b) 3D printed multi-scale spider web displaying interacting radial and spiral elastomeric filaments [32]. (c) 3D printed multi-scale biomimetic artificial skin [33]. (d) Bio-Inspired Vapor-Responsive Colloidal Photonic Crystal Patterns by multi-scale inkjet printing [34]. (e) The multiscale printing based on scanning projection stereolithography and the 3D printed lattice structures [35]. (f) A schematic diagram of multi-scale fabrication process. The multi-scales in the XY plane are achieved by using laser beams in different sizes, and the multi-scales along the $Z$ axis are realized by using different layer thicknesses [36]. (g) 3D-printed eagle eye: compound microlens system for foveated imaging [37].

\subsection{MULTI-SCALE AM PROCESSES}

With increasing researches on the design of multi-scale structures for different functions, the fabrication of novel multi-scale system has become important. The development of proper additive manufacturing process for multi-scale design is desired and critical to achieve certain functionality. However, most of existing AM technologies can only fabricate 3D structures with a uniform resolution throughout the entire printing process. In fact, an amount of functional structures contains micro and/or nano scale features on macro scale subtract, such as shark skin, peristome surface of Nepenthes alata, gecko feet, fly eyes and butterfly wing etc. (ref. [38]). It is difficult for the traditional manufacturing processes to fabricate such complex geometric shape, and the functional performance of built artificial biomimetic object cannot be comparable with respect to their genuine counterparts in nature. Therefore, the development of multi-scale AM processes attracts much attention in many recent researches. There are two main representative ideas that have been investigated. One solution is to fabricate multi-scale structures by the integration of multiple AM processes, each of which best suits a certain scale. This method can make 
multiple sections of a structure by applying optimized processes [39, 40, 41], and the hybrid process can provide capability and flexibility superior than operating each individual printing process separately. However, the integrated printing solution only adapts to build multiscale 3D objects with a special design, while how to integrate different printing processes to fabricate objects with any universal design is still a significant challenge. Another solution that has been investigated is the standalone printing process that is capable to achieve multi-scale fabrication on its own.

\subsubsection{Stereolithography (SLA) based Multi-Scale 3D Printing}

To generate smooth and uniform micro-scale structures, liquid writing needs to be highly accurate thus requires high-precision motion control of the printing nozzle. SLA process, in contrast, can print high resolution micro features using easily controlled light exposure [42, 43]. However, the size of macro-scale focus image, which in turn determines the fabrication range, is restricted to the physical dimension of DMD chip, and such issue is also open to be resolved for the purpose of multi-scale fabrication [40]. One straightforward solution is to joint multiple DMD chips to shape a larger array, but the uniformity of light becomes hard to control with multiple DMD chips working at the same time. Other than that, multi-process or hybrid process could be a potential solution to address this problem. For example, a swirling flow coaxial phacoemulsifier sleeve with internal micro-vanes were fabricated by combining multi-jet modeling(3DP) and micro-mask image projection based micro-stereolithography (PuSL) [39]. Besides, some multi-scale structures, whose dimension ranges from macro scale to nano-scale, were fabricated by the integration of SLA process and two photo polymerization process [40]. Alternatively, in the case of mask image projection (MIP) based SLA process, researchers also achieved multi-scale fabrication by adding movement of projection light beam. Emami [44] etc. extended the fabrication area of MIP based SLA process by moving the optical system only one-pixel length each step, illuminating one portion with several times exposure. The known issue for this method is that overlaps exist between two adjoining printed sections [44, 45]. To eliminate the overlap, as shown in Figure 2(h), Lee et al. [35] developed a low-cost scanning projection printing system, which can project micro-scale features at $10 \mathrm{um}$ onto a $5 \mathrm{~cm}$ large area by precisely controlling the exposure time.

Meanwhile, laser spot with dynamic changing focus can also be used to print multiscale structure [46]. This means that, the laser spot was selected respectively to cure corresponding sizes of features. Similarly, laser can be adjusted by optical filter with high-contrast gratings to accommodate features at different scales, similar to the principle of nano lithography technology [47]. However, it is time-consuming to fabricate large area objects using nano-scale laser beam. To solve this problem, Mao et al. [36] proposed an optimized multi-scale fabrication process with shaped beams, and the printing speed and resolution were improved dramatically. The process planning associated with the shaped beam method is as illustrated in Figure 2(f). SLA-based multi-scale process is applied to fabrication cases that are either hard or even impossible to implement. Multicomponent objective lenses mimicking the eagle eye was designed and printed by SLA process onto a complementary metal-oxide semiconductor (CMOS) image sensor [37]. This artificial optical system shown in Figure 2(g) can achieve a full field of view of $70^{\circ}$, with the angular resolution up to 2 cycles/deg in the center of the image [37, 48]. Besides, micro- and nano-optics with complex artificial eye lens was fabricated by a novel micro-scale 3D print method called femtosecond two-photon direct laser writing. The printed micro-lens at $100 \mu \mathrm{m}$ forms a high-performance multi-lens with the field of view at $80^{\circ}$.

\subsubsection{Other Multi-Scale 3D Printing Methods}

Multi-scale fabrication can be implemented by 3D printing with nozzles in different sizes. As show in Figure 1(a, b) and Figure 2(a, b), large scale structures with micro-scale features were successfully fabricated by the method of direct ink writing, where the fabrication resolution can be controlled by multiple 
parameters, such as the moving speed of nozzle, the material feeding speed and the dimension of nozzle $[49,50,31,51,52,53,54]$. Similarly, a new direct ink writing method was developed to fabricate multiscale biologic structures, e.g., micro vascular network. In the process shown in Figure 2(a), hydrogel was injected into the gel tank according to the 3D geometry of vascular structures, followed by the forming of vessel's cavity as injected hydrogel turned to be liquid when temperature changes [31]. Besides, multi-scale complex fluidic networks were printed in granular gel slurry based on nozzle printing [50]. After removing granular gel from the printed structures, multi-scale hierarchical branching networks were easily generated with the dimension ranging from $100 \mathrm{um}$ to $10 \mathrm{~mm}$. Recently, a nozzle-based printing process was applied to fabricate a multi-scale biomimetic, spiders spin that possesses high strength, elasticity and tensile failure stress at the same time. As shown in Figure 2(b), the mechanical response of elastomeric webs was investigated under multiple loading conditions, and results showed promising characteristics of the performance [32].

Recent progress on multi-scale AM technologies simultaneously facilitates the performance study of multi-scale structures, such as superhydrophobicity, self-cleaning, drag reduction, energy conversion, biological self-assembly, focusing imaging etc. [55] As an example, special placoid scales associated with the surface of shark skin has intriguing property to reduce frictional fluid drag dramatically. Such special placoid scales, which are difficult to build by using traditional manufacturing technologies, have been successfully built onto the artificial shark skin by the nozzle based 3D printing methods shown in Figure 2(c), and such multi-scale shark skin presented excellent flow ability compared with the surface without any micro features [33]. Also, multi-scale nozzle-based printing process provides a great promise to develop advanced optical elements, such as dynamic display or multifunctional sensor array. For example, multicolor shifting van was printed with multi-scale colloidal photonic crystals patterns using mesoporous colloidal nanoparticle ink, as shown in Figure 2(d). Through adjustment on the size and mesoporous proportion of nanoparticles, original color and vapor-responsive color shift were precisely and easily controlled [34]. While 3D printing being introduced to more applications of biology, tissue engineering, autonomous vision and optical systems, the multi-scale capability development of $3 \mathrm{D}$ printing continues to interest researchers and industry professionals.

\subsection{SHAPE CHANGING AM PROCESS DEVELOPMENTS}

Tibbits et.al [56, 57] used a term four-dimensional (4D) printing to describe a class of 3D printing technologies applied to build shape-changing structures (see Figure 3(a)). 4D printing overcomes the traditional fabrication limitations by designing heterogeneous materials to enable the printed structures evolving over time (the fourth dimension) $[58,59,60,61,62,63]$. There are many different ways to generate the time-variant change using 3D printing process, we list the most common approaches in this section.

\subsubsection{Thermal Stimuli}

One way to build the shape changing structure is by using the process of multi-material printing and thermal stimuli $[24,64,65]$. Basic shape-changing materials available for these multi-material 3D printing techniques include shape-memory polymer (SMP) [66] and hydrogel [67]. Elastomers are typically used as the passive matrix in these structures. Ge et al. [58] printed active composite materials realized by directly printing glassy SMP fibers in an elastomeric matrix (see Figure 3(b)). The initial configuration is created by $3 \mathrm{D}$ printing, and then the programmed action of the shape memory fibers creates time dependence of the configuration (i.e., the 4D aspect). The printed structure can be thermomechanically programmed to assume complex three-dimensional configurations including bent, coiled, and twisted strips, folded shapes, and complex contoured shapes with non-uniform, spatially varying curvature. More interestingly, incorporating more than one shape-changing material with different responsive properties enables sequential shape changes via the same multi-material 3D printing techniques. For example, when multiple 
SMPs with different thermomechanical properties were incorporated, time-dependent sequential shape changes can be accomplished by changing the triggering temperatures, as shown in Figure 3(g) [24]. A 3D printed gripper was opened (closed) after programming and the functionality of grabbing (releasing) objects was triggered upon heating. Deng et al. [68] present 4D printing of self-folding structures that can be sequentially and accurately folded (Figure 3(h)). When heated above their glass transition temperature, prestrained polystyrene films shrink along the XY plane. Silver ink traces printed on the film are used to provide heat stimuli by conducting current to trigger the self-folding behavior. Programmable structures such as a lock and a three-dimensional antenna are demonstrated to illustrate the feasibility and potential applications of this method.

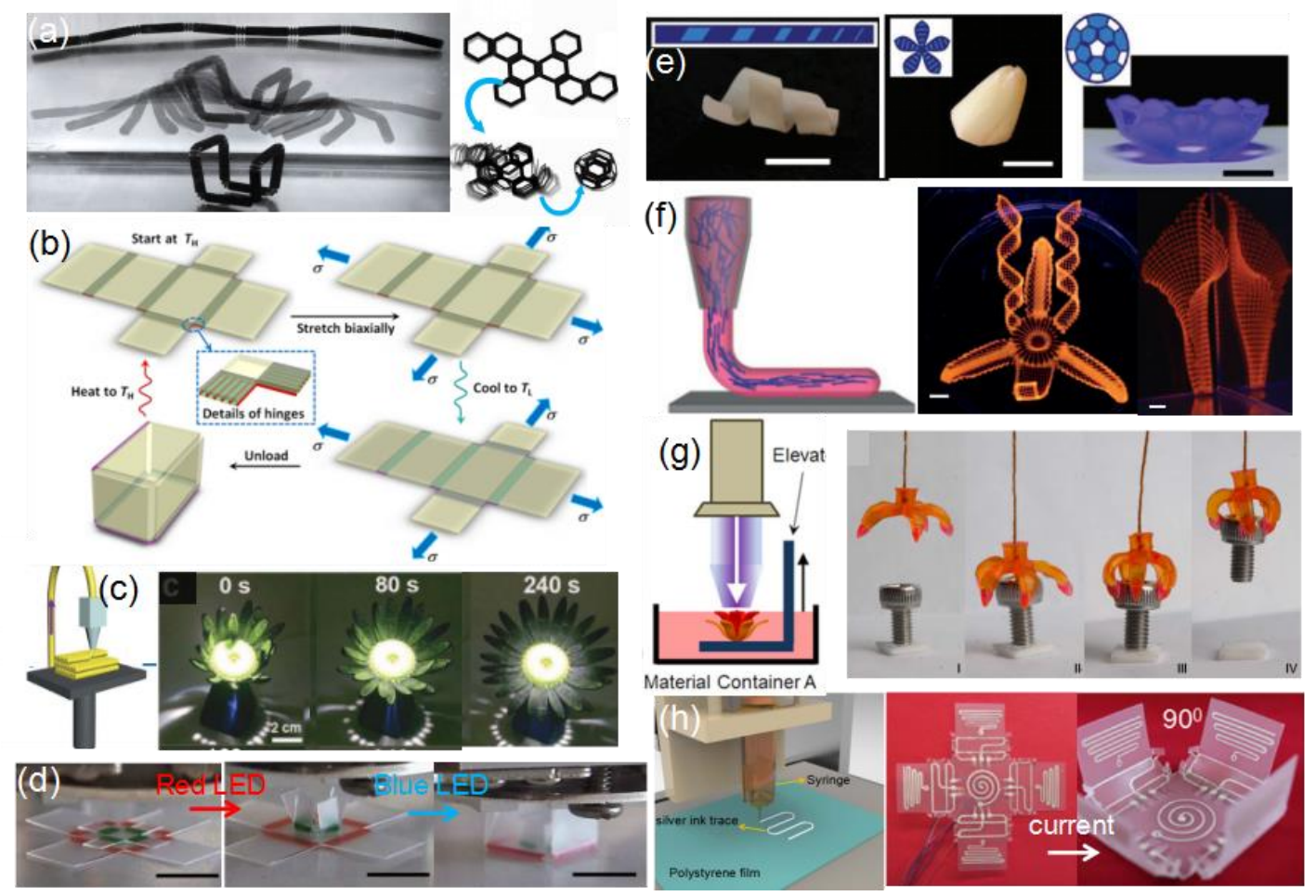

Figure 3 (a) Photographs show the transformation from a flat 3D printed structure to a precise folded structure [56, 57]. (b) A self-folding and opening box fabricated by $4 D$ printing [58]. (c) A 4D printed photoresponsive flower [69]. (d) Sequential selffolding nested boxes - a small box with green hinges on top of a large one with orange hinges [70]. (e) Self-folding structures with cross-link density gradient achieved by controlling light exposure [71]. $(f)$ Biomimetic $4 D$ printing structures -fabricated by direct ink writing with shear-induced fiber alignment [72]. $(\mathrm{g})$ The snapshots of the process of grabbing an object by multi-material $4 D$ printing [24]. (h) A self-sequential folding antenna structure utilizes 4D printing [68].

\subsubsection{Light Stimuli}

Light stimuli is also used to build shape changing structures. Yang et al. [69] demonstrated the 3D printing of photoresponsive shape memory devices through combining FDM printing technology and photoresponsive shape memory polymers/carbon black composites (see Figure 3(c)). A biomimetic sunflower is built to demonstrate its shape changing function under sunlight. Furthermore, Liu et al. [70] even created a sequential folding structure by different color of light sources (see Figure 3(d)). Printed ink on the surface of the polymer sheets can discriminately absorb light on the basis of the wavelength and the color of the ink that defines the hinge about which the sheet folds. The absorbed light gradually heats the underlying polymer across the thickness of the sheet, which causes relief of strain to induce folding. These color patterns can be designed to absorb only specific wavelengths of light, thereby providing control of sheet folding with respect to time and space. This type of programmed shape variation can have numerous 
applications, including reconfigurable electronics, actuators, sensors, implantable devices, smart packaging, and deployable structures.

\subsubsection{Moisture Stimuli}

On the other aspect, the anisotropy in a 3D printed structure is used to trigger shape changing by moisture. The mechanical anisotropy comes from bio-inspiration can be realized by various methods including magnetic alignment and shear force alignment of fillers [73, 74, 75, 76, 77]. The cross-linking density gradient in polymers can be achieved by tuning the process parameters during the fabrication process, such as the light dose exposure in SLA, or the heating temperature and the moving speed of the nozzle in FDM [71]. The shrinkage is constrained by the building platform during the fabrication process, yielding a strain gradient within the 3D printed structures (as shown in Figure 3(e)). Heating the final structures releases the built-in strain and accordingly results in desired shape-changing behaviors. Shearinduced alignment has been successfully used in a direct ink writing process to attain localized swelling anisotropy in swellable shape-changing structures, as depicted in Figure 3(f) [72]. The alignment of stiff cellulose fibrils in hydrogels was controlled by the prescribed printing paths and the extent of the alignment can be adjusted by the nozzle diameter and the printing speed. After being cured by UV light, a printed structure was immersed in deionized water to initiate the swelling-related shape changes. Compared with the traditional subtractive manufacturing processes to fabricate fiber-based hydrogel composites [78], the 3D printing technique offers more flexibility in controlling the local alignment of fibers during the fabrication process, which enables the shape-changing structures to have more complex behaviors.

Overall, shape-changing structures can be achieved via 3D printing responsive and deformable materials in a bio-inspired architecture with anisotropic material properties. The responsive and deformable materials can exhibit simple shape changes, such as shrinkage or expansion, upon exposure to external stimuli including moisture, heat or light.

\subsection{MULTI-FUNCTIONAL AM PROCESSES}

AM processes are further used to build multi-functional structures (e.g., multifunctional flexible sensors $[79,80,81,82,83]$, electronics $[84,85]$ and hydrodynamic structures [86]). Given the advantage of being able to fabricate objects with minimal limitation on geometry, AM technologies have already been used to enhance functionality or performance without rapidly increasing the cost of fabrication.

A multi-material, multi-scale, and multi-functional 3D printing approach has been employed to fabricate 3D tactile sensors under ambient conditions conformally onto free form surfaces [87]. The customized sensor is demonstrated with the capabilities of detecting and differentiating human movements, including pulse monitoring and finger motions. The 3D printed tactile sensor consisting of a base layer (silicon), top and bottom electrodes (with 75\% Ag/silicon), an isolating layer, a sensor layer (with 68\% $\mathrm{Ag} /$ silicon), and a supporting layer (see Figure 4(a)). The printed flexible, stretchable, and sensitive sensors were found proven to be capable of detecting and differentiating human movements, including radial pulse, and finger pressing and bending in [88]. Development of a custom-built multifunctional 3D printing process, combined with functional inks, is at the core of this approach and determines the features of the final devices. A real-time monitoring system of body motion was designed by using smart phones and 3D printed CSH hydrogel. The autonomous intrinsic self-healing of the hydrogel is attained through dynamic ionic interactions between carboxylic groups of poly(acrylic acid) and ferric ions (Figure 4(b)). Establishing a fair balance between the chemical and physical cross-linking networks together with the conductive nanostructure of polypyrrole networks leads to a double network hydrogel with bulk conductivity, mechanical and electrical self-healing properties (100\% mechanical recovery in $2 \mathrm{~min}$ ), ultrastretchability $(1500 \%)$, and pressure sensitivity. 3D printing is used for fabricating programmed bacterial cells into large- 
scale $(3 \mathrm{~cm})$ high-resolution $(30 \mu \mathrm{m})$ living networks that accurately respond to signaling chemicals in a programmable manner (Figure 4(e)). Novel applications enabled by 3D living printing of programmed living cells were demonstrated in [89], including logic gates, spatiotemporally responsive patterning, and wearable devices. The integrative technology of 3D living printing has the potential to be used as a general platform where a range of genetically programmed cells, matrices, and structures can be applied to design more customized living materials and devices with predictable dynamic functionalities.

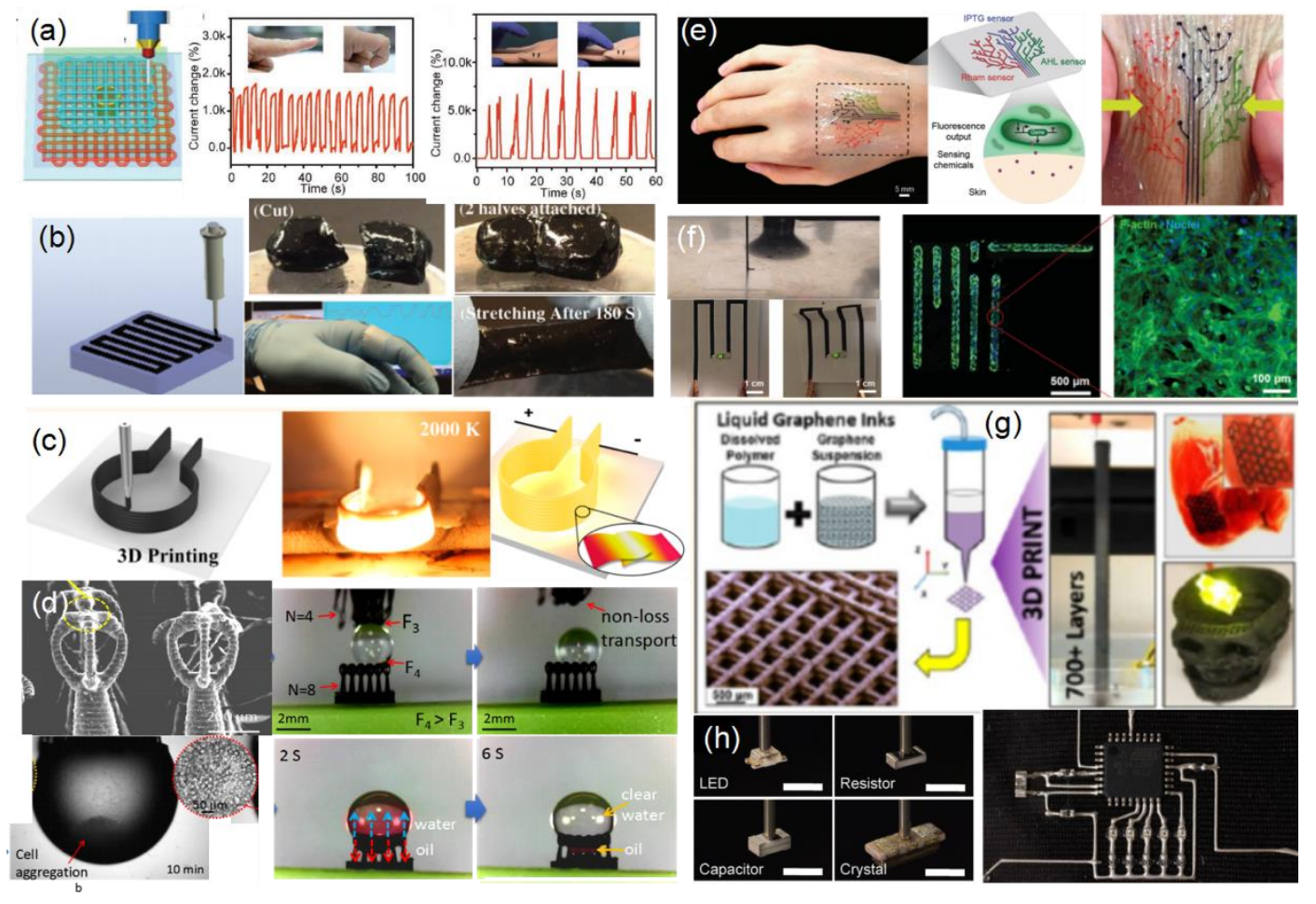

Figure 4 (a) 3D printing of multifunctional sensor with helicoidal structures for bending and tactile sensor [87]. (b) 3D printing of flexible sensors with self-healing hydrogels [88]. (c) 3D printed high temperature and high rate heater [90]. (d) 3D printed biomimetic multifunctional superhydrophobic microstructures for non-loss microdroplet transportation and oil/water separation [9]. (e) 3D printed living tattoo for chemical detection on human skin [89]. (f)3D printing of bioactive carbon nanotube-based ink for flexible electronics [91]. (g) 3D printed multifunctional user-defined architectures from graphene inks for electronic and biomedical applications [92]. (h) Hybrid 3D printing of soft electronics, image of pick and place of surface mount electrical components, including LED, resistor, capacitor, crystal oscillator and microprocessor chip [93].

Yao et al. [90] developed 3D printed reduced graphene oxide (RGO)-based heaters to function as highperformance thermal supply with high electrical conductivity, high temperature and ultrafast heating rate. Joule heating was used to effectively reduce RGO at high temperature (Figure 4(c)). The resulting 3D heater with RGO flakes can reach a high heating temperature, up to $3000 \mathrm{~K}$. The heater temperature can be ramped up and down with extremely fast rates (i.e., up to about $20000 \mathrm{~K} /$ second). The 3D printable RGO heaters with different shapes can be applied to a wide range of nanomanufacturing when precise temperature control in time, placement, and the ramping rate are important. Superhydrophobic microstructure inspired by Salvinia molesta [9] was fabricated by the immersed surface accumulation 3D printing process. The multi-scale artificial hairs with eggbeater heads were reproduced according to the eggbeater structure design in nature. The head is fabricated by intersecting different number of circumferences with a diameter of $35 \mu \mathrm{m}$ and a height of $250 \mu \mathrm{m}$ (Figure 4(d)). The controllable adhesive force (from $23 \mu \mathrm{N}$ to $55 \mu \mathrm{N}$ ) can be easily tuned with different number of eggbeater arms. The results show that the 3D-printed eggbeater structure could have numerous applications, including 3D cell culture, water droplet manipulation, micro reactor, oil spill clean-up, and the separation of oil/water mixtures. 
Shin et al. [91] successfully developed electrically conductive CNT-based inks that were cytocompatible by using bio-surfactants including DNA, HA, and chemically modified gelatin to successfully improve CNT dispersion in water and its stability (Figure 4(f)). The developed electronic circuits possess good maintenance of the resistance as well as good cellular behavior. 3D printed circuits were built with bio-ink and embedded within hydrogel constructs. The developed CNT hybrid materials will be beneficial for fabrication of flexible and foldable biosensors and advanced functionalized tissueengineered scaffolds. A 3D printable graphene (3DG) composite consisting of majority graphene and minority polylactide-co-glycolide, as a biocompatible elastomer, is 3D-printed from a liquid ink (Figure 4(g)) [92]. In vivo experiments indicate that 3DG has promising biocompatibility over the course of at least 30 days. Surgical tests using a human cadaver nerve model also illustrate that 3DG has exceptional handling characteristics and can be intraoperatively manipulated and applied to fine surgical procedures. This 3D printable composite could be applied toward the design and fabrication of a wide range of functional electronic, biological, and bioelectronic medical and nonmedical devices.

Hybrid 3D printing is a new method for producing soft electronics that combines direct ink writing of conductive and dielectric elastomeric materials with automated pick-and-place of surface mount electronic components within an integrated additive manufacturing platform (Figure 4(h)) [93]. Using this approach, insulating matrix and conductive electrode inks are directly printed in specific layouts. Passive and active electrical components are then integrated to produce the desired electronic circuitry by using an empty nozzle (in vacuum-on mode) to pick up individual components, place them onto the substrate, and then deposit them (in vacuum-off mode) in the desired location. The components are then interconnected via 3D printed conductive traces to yield soft electronic devices that may find potential application in wearable electronics, soft robotics, and biomedical devices.

The growing mature of multi-material and multi-scale printing capabilities enabled different industries to develop new products that are tailor-made, high performance and multifunctional. We will see much more radical change in the way that the products are designed and manufactured. At the same time, the needs for standardized and automated design process are becoming bigger and bigger, and computational design techniques are one of the most essential components of additive manufacturing.

\section{RECENT DEVELOPMENT ON DESIGN FOR AM TECHNOLOGIES}

AM processes always start from a three-dimensional (3D) solid model as input, which specifies the necessary information for fabricating the exact shape we need. Usually, only geometry is provided in a digital CAD file and is converted to layers by the slicing software. The layers hereby depict the contour of the model in a way that facilitates the layer-by-layer printing processes. Researches have been conducted for decades to enhance the quality and the complexity of the fabricating results [13].

Recently, a paradigm shift has occurred in the field of additive manufacturing, from the geometrycentered fabrication to support functional needs with the emergence of new printing processes. This shift is changing the design methodology from single requirements to cater the solution for multiple requirements such as fabricating heterogeneous objects to enhance mechanical properties. Additional information like materials are needed to be processed in the design stage.

In this section, we review the current design methodologies and discuss the challenges for each type of AM processes.

\subsection{DESIGN FOR MULTI-MATERIAL}

Multi-material object often refers to the class of objects that have different materials composition. Although state-of-the-art CAD software is capable of defining discrete multi-material regions, the variety 
in functional requirements, materials and processes still makes the formulation of a standard pipeline challenging. Most of the CAD software only focuses on how to assign the materials, such as through geometry operations [94], yet lacks the ability to design where to place the materials to achieve demanded properties. The following sub-sections will explore the variety of design methodologies for heterogeneous objects - specific about enhancing structural and material properties by fabricating an object through multimaterial AM processes.

\subsubsection{Periodic Heterogenous Objects}

Periodic cellular composite is one of the material classes that benefits from the new technology in controlled composition, geometric shape and complexity. The composite structure consists of many identical base cells or representative volume elements (RVEs), and each cell incorporates structural material (matrix) and reinforcements (e.g. fibers). There are many different multiphase composite materials that exist in nature or being synthetically fabricated. They are distinguished from discrete and continuous manners. The former (see Figure 5(a)) is reinforced with dispersed particles or fibers (e.g. continuous or discontinuous carbon fibers, carbon nanotubes and graphene, etc.) that characterizes the performance and properties through manipulating fiber volume fraction, fiber aspect ratio and fiber orientation [3]. The latter (see Figure 5(b)) has phases that are interconnected and co-continuous in the cell [95]. This class of shapes is represented by triply periodic minimal surfaces (TPMS), in which the surface is described by a mathematical function, allowing designer to easily architect the topology and arrangement of the constituents toward desirable isotropic or anisotropic mechanical/physical properties.

(a)
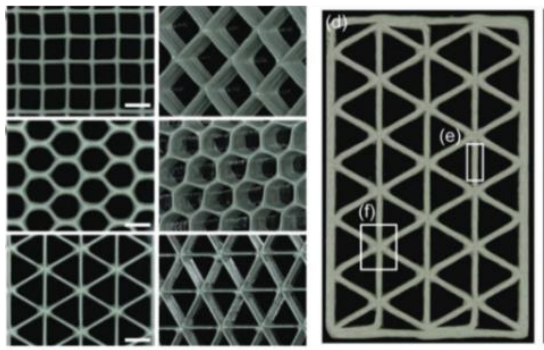

(c)
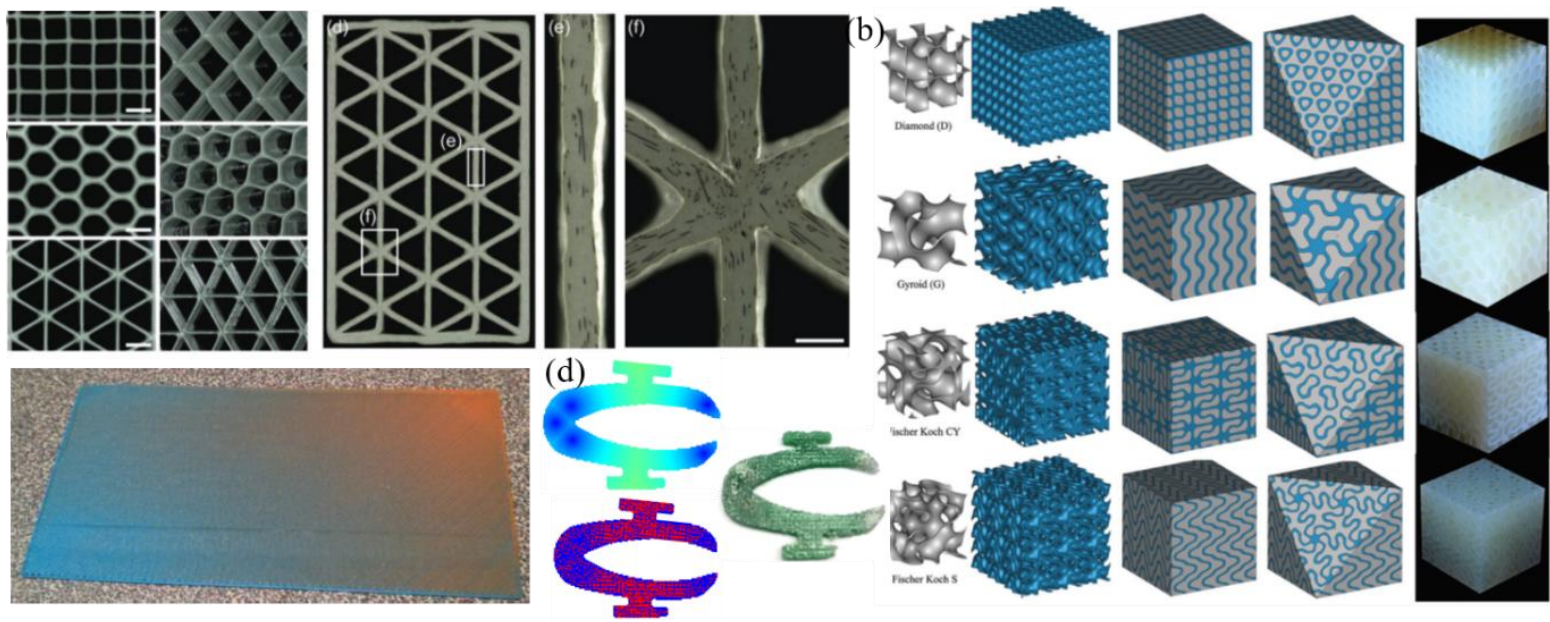

Figure 5(a) Discrete cellular composite in square, hexagonal and triangular honeycomb structures. The triangular honeycomb structure is printed with highly aligned carbon fibers oriented along the print direction [3]. (b) Different TPMS topologies used as composite solid [95]. (c) An example of gradient structure [96]. (d) An example of using two materials to approximate the deformation of continuous material distribution. The blue-to-red colors represent the Young's modulus. The simulation and the fabrication results demonstrated the effective of their approach [97].

Design methodologies for discrete cellular composites can be classified into two main groups: intuitiondriven design [2] and topology optimization [98]. Compton et al. [3] created lightweight cellular composites with controlled alignment of fiber reinforcement inspired by balsa wood. Yang et al. [5] and Gu et al. [4] designed different architectures that are inspired by lobster claw and nacre respectively, to enhance the impact resistance of the composite. Computational (finite element) models are often used to characterize the performance and properties of the designed composites, taking into account the material's properties [99]. Hu et al. [2] proposed to randomly distribute the reinforcement to extract properties of the composites. Quan et al. [100] reviewed other design models of reinforcement for cellular composite, such as woven 
fabric and braided preforms. With computational modeling and simulation, optimization of high performance design would be possible. However, when building composite involves several groups of intertwining yarns, such as textile assemblies, the computational cost on existing CAD design tools could be extremely high.

To obtain the optimized design of cellular materials, some recent works have focused on topology optimization. For instance, Long et al. [101] proposed a concurrent optimization for composites composed of phases with distinct Poisson's ratio. Their approach optimized the composite unit cells and their distribution in macrostructures simultaneously. Nevertheless, new frontier AM technologies keep increasing the printing dimension of the objects, and concurrently the available printing resolution becomes finer. As a result, the computational effort rapidly becomes prohibitive.

Another composite material, periodic interpenetrating phase composites (IPCs), has recently caught attention due to its smooth-curved nature of the TPMS surfaces, which possesses higher surface area to volume ratio. As a result, it provides exceptional mechanical, thermal, acoustic and electrical properties [95]. There are two variants of TPMS solid of composites. One is sheet solid (created by thickening the surface) where the volume fraction is controlled by the desired thickness. Another one is network solid (solidify one phase of the surface) where the volume fraction is controlled by the approximated level-set constant. Several studies showed that sheet solid possess better mechanical properties than the network solids at the same volume fraction [95, 102]. Design methodologies for modeling TPMS solids have been proposed in the literature [103, 104, 105], which mainly adopt volume representation (voxels or volumetric distance field) to describe the model. Last but not the least, with the help of multi-material technology, the field of metamaterials [6] has been extended from homogeneous to heterogeneous. Additional materials in metamaterials offer a different way to increase one specific property without compromising in other properties. For example, a stiffer beam means designing a thicker beam, which lowers the flexibility of the joints. Because of the underlying contradictions in single material design, using additional materials can tune the properties and overcome the geometry barriers $[7,106]$. Unfortunately, currently available CAD software are limited in their ability to provide fully interactive design function for heterogeneous periodic structures. The difficulties come from the inability to assemble unit cells as to form a complex scaffold and efficiently represent internal architecture in terms of materials. To relieve the burden researchers have developed geometric representations and utilized GPU to offload some computationally intensive tasks [107].

\subsubsection{Graded Heterogeneous Objects}

Functionally graded material (FGM) can be characterized by the variation in composition and structure gradually over volume, resulting in corresponding changes in the properties of the material. FGM as an interface layer that combining two or more materials in the same component (Figure 5(c)), such as metal and ceramic, enhancing the bond strength [108] and effectively overcome the shortcomings of traditional composite material [109].

Designing FGM objects is difficult because the distribution of internal material cannot be simply bounded by a geometrical shape. Additionally, functional outcomes are very important and should be considered during the design. Important aspects for FGM design include model representations, process planning and evaluation of material properties [110]. Different representations have been proposed in literature [111], which can be classified into two main groups: discretized representations and functionbased representations. The discretized model (e.g. distance field) can specify the composition of every volumetric element within the parts, but it requires enormous amount of data and designing methods are not intuitive. Function-based models use a global function or piecewise blending functions in different 
regions to represent mathematically the volume fraction composition of the part at each point. Yet, for complex geometries, this representation often does not work well, especially when retrieving material at specific location. In order to generate a fully optimized FGM part, optimizing both topology [112] and material composition [113] on suitable representation is necessary. On the other hand, researches show that a particular AM process chosen to make the part can significantly affect a design's outcome. Hence, manufacturing constraints should be associated with the representation, allowing the optimal parts to be adjusted based on process planning strategies - include slicing, orientation and path planning [110, 96].

The concept of FGM can be further integrated with microstructure/porous structure. Bahraminasab et al. [114] designed a new metal-ceramic porous functionally graded biomaterial to replace the existing metal alloy material normally used. The use of metal-ceramic FGM reduced the stress-shielding effect of the femoral component, which is the primary cause of aseptic loosening. And the porous structure can cause more uniform stresses in the femur. This type of new material is particular useful in bio-engineering applications, as the porosity structure facilitates the cell activities. To fully make use of the integration concept, optimization and design methodologies for the constituents' material gradation, interface geometry and porosity in the structure need to be further studied [115].

\subsubsection{Non-Periodic Heterogeneous Objects}

Most non-periodic heterogeneous designs are goal-driven with respect to different functionality. Brunton et al. [116] produced 3D color prints which are highly accurate and detailed with 4 translucent materials. They developed an error-diffusion algorithm on voxel representation of surfaces to approximate the color gradients. Gu et al. [117] proposed an algorithm to yield designs that compose of soft and stiff material to create composites with more than 20 times tougher than the stiffest single material used in the composite. The authors optimized the design using a modified greedy algorithm. The algorithm works by picking an initial random geometry and then switching all elements one by one and checking if the stiffness can be improved. The authors later proposed a machine learning method to improve the accuracy and efficiency [118]. Bader et al. [119] presented a data-driven approach for the creation of high-resolution, geometrically complex, and materially heterogeneous 3D printed objects. This approach utilized external data source as the primary design element and can generate material distributions during slicing. The models derived from these examples rely heavily on a discrete representation to describe the whole solid point by point. But they often have problem to be scaled for the production of high-resolution and/or largevolume model. On the other hand, standard techniques like topology optimization do not scale well and they cannot be run on objects with billions of elements (or voxels) due to the large number of design variable. Therefore, Yu et al. [120] proposed to design and perform optimization on model in meso-scale. The basic idea is to optimize the design in coarser level and then further optimize with finer scale via finite element analysis. Leung et al. [97] proposed a data-driven approach to design composite structure (soft and stiff materials) such that it can achieve prescribed deformation. They discretized the design domain and approximated a solution by finding composite patterns that possess closely matched behavior (Figure 5(d)). Kennedy [121] also presented a work to address the difficulty of large-scale. He proposed to use multigridpreconditioned Krylov method for solving large structural finite-element problems and a parallel interiorpoint optimization technique for solving large-scale constrained optimization problems.

To represent a multi-material object, most geometric-based representations are insufficient to specify material composition, especially when the material distribution needs to associate with the functional specification. Simply integrating both geometric and material information in one representation (e.g. voxels) may not be a good option due to the redundancy and can't be design intuitively, although it is favorable for

the functional analysis. Developing a representation that can work in both design and analysis stage could be the best use for multi-material printing [122]. 


\subsection{DESIGN FOR MULTI-SCALE AM PROCESSES}

As emerging AM technology can fabricate details of materials at multiple scales to achieve behaviors significantly differing from the $3 \mathrm{D}$ printing material as solid, the next generation of CAD system needs to support specification at multiple scales. However, the design methodology and representation could be significantly different among the scales. For example, the reverse engineering in macro-scale is normally based on 3D surfaces, while it is mainly based on digital material images obtained from microscope scanning in nano-scale. The challenge is to link the various scale transitions or to model both the geometry and material structure concurrently. As mentioned by Panchal et al. in a review paper [123] that a transition method may only be applied to some scales. Their paper focuses on the micro- to macro-scales for AM applications. We will review the design strategies and different representations for multi-scale modeling and printing.

\subsubsection{Biomimicry and Topology Optimization}

It is found that the major factors of the structures affecting the properties are the topology, shape and density [124], besides the material itself. Therefore, much progress has been made in developing and identifying microstructures with desired behavior [125, 126, 127]. Structures at the scale of microns influence the physical properties such as weight and ductility. Micro-structured material can be considered as a new material [128] with totally different properties compared to its primitive as a solid. To design the microstructures, an intuitive approach is to mimic the forms found in the nature such as bone (Figure 6(a)) and foam (Figure 6(b)). By taking X-ray tomography or scanning electron microscope images, Vlasea et al. [129] took the natural bone porous macro architecture as an input, and draw the micro-channels to mimic its biological and mechanical properties. Quinsat et al [130] extracted surface information from the images and represented the information by skin voxels corresponding to the internal and external surfaces in subvoxel to super-voxel scales, which are used to determine the filling strategy. Martinez et al. [131] applied the Voronoi diagram to generate an implicit representation to create foam geometry. The procedural Voronoi foams are the microstructures to exhibit different elastic behaviours. The implicit modeling adapts locally to follow the elasticity field but connect the frame and microstructures seamlessly. Martinez et al. [132] synthesized the foam structures by controlling the elasticity independently along three orthogonal axes, called orthotropic foams. The fine-scale structures are generated procedurally and resemble a foam, which can be scaled to arbitrarily volumes.

The mathematical way to optimize the microstructure and material layout for given loads and boundary conditions in a design domain is topology optimization (TO) (Figure 6(c)). TO is one of the early approaches developed for microstructure design, such as homogenization method [133], solid isotropic material with penalization (SIMP) [134], differential growth [135, 136] and level-set method [137, 138]. Several CAD software, such as DreamSketch, Solidworks and SolidThinking Inspird, have been developed to generate design using topology optimization [139, 140, 141]. However, these are iterative approaches which perform FEA repeatedly until the best solution is found, resulting in high computational efforts, especially in fine resolution of design domain. Another widely used approach, called Ground Structure [142, $143,144,145,146]$, which starts with the union of all potential members, and eliminates the "vanishing" ones, i.e., zero cross-section area, throughout the optimization process. Gilbert and Tyas [147] introduced a growth method called "adaptive ground structure", which starts with an initial, minimal connectivity, ground structure, and increases the number of members at each step. This approach is further modified by Sokół $[148,149]$ in a level-set manner by first considering the shortest members in the ground structure, and then longer members are considered as candidates in each next iteration. Wu et al. [150] speeded up the voxel-based TO by aggregating local per-voxel constraints to global constraint, and the method can work on high-resolution design domain. Kwok et al. [151] made use of principal stress lines to convert the 
optimization to a geometry design problem, and their method is less dependent on the resolution of finite elements and can achieve interactive speed. To solve the problem of support removal for infills, TO is computed on a carefully designed rhombic structure so that the self-supporting can be guaranteed on the result of optimization [152].

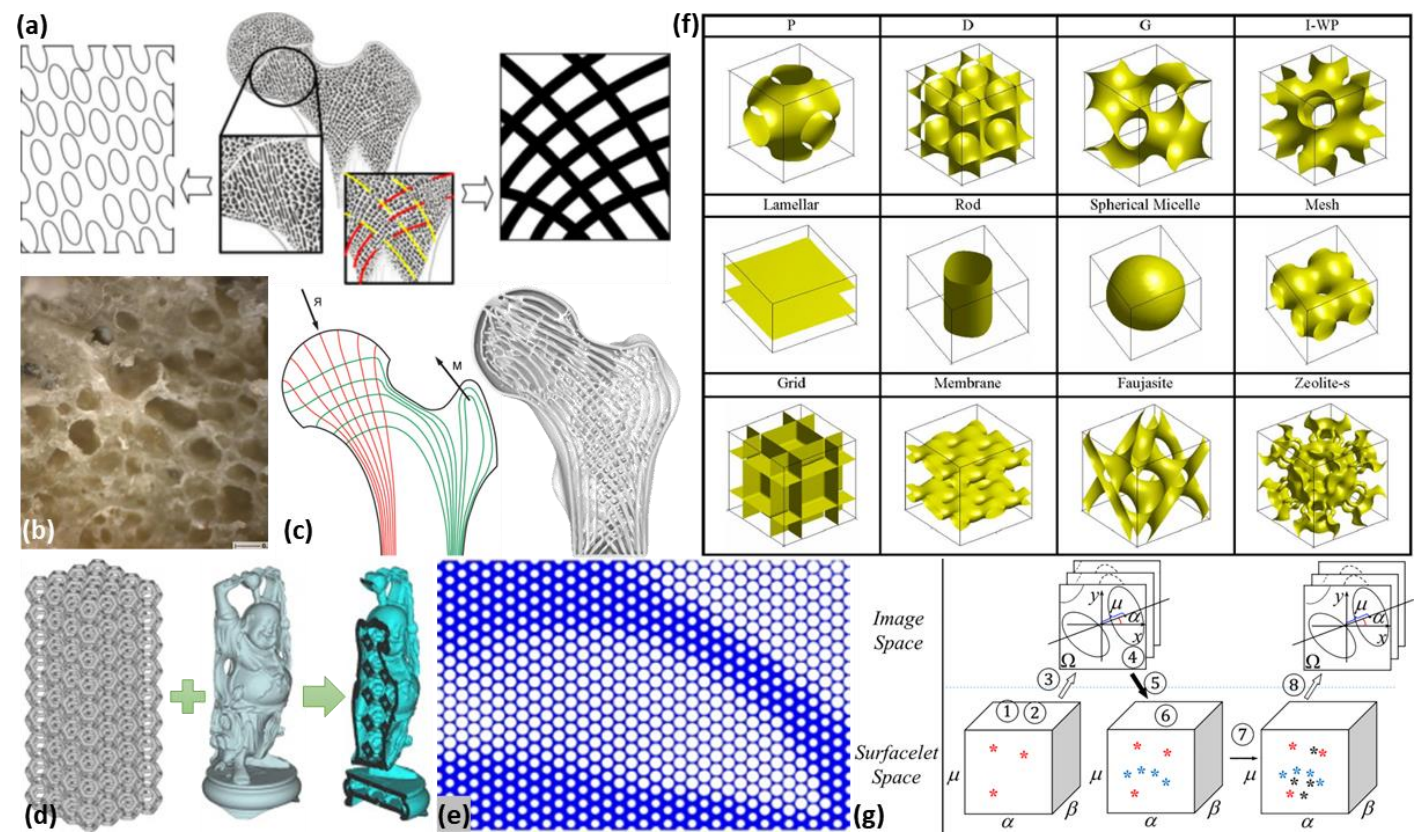

Figure 6 (a) Natural bone architecture is mimicked by integrating network of channels and pores [129]. (b) A printed structure with sugar particles dissolved mimicking natural foam structure [153]. (c) Topological optimized porous infill in a bone model [150]. (d) Surface and internal structures can be modeled by Boolean operation [107]. (e) A pseudo periodic cellular structure designed by cells with same topology but different sizes and shapes [154]. (f) Cells defined by geometry function [155]. (g) The specification of material function can be designed by transforming between image and surfacelet spaces [156].

\subsubsection{Cellular Structure}

Although the concurrent methods mentioned above designing the geometry and material at the same time, it can only explore a very small region of the design space because of its computing cost. When the structures need to fill large volumes, the current computational power and memory are not sufficient to manage. Rosen [157] proposed using manufacturable elements as an intermediate representation to support manufacturing. The manufacturable element is a predefined and parameterized decomposition of a volumetric region of a part, like a cell. The periodicity simplifies modeling and simulation, reduces memory costs, and can be assembled by Boolean operation [107] (Figure 6(d)). The cellular structure is promising in a variety of engineering applications. Sá et al [158] generated a parameterized infill cellular structure adaptively by a recursive subdivision approach. Given a volume boundary, this approach makes use of primal or dual cellular structure to fill up the volume, which can be 3D-printed. Qi and Wang [159] presented a feature-based approach to create crystal structures with such building blocks, where periodic surfaces of basic features are used to construct complex crystal structures rapidly and parametrically. Xiao and Yin [160] modeled the random structure of porous media using Voronoi tessellations.

Lattice structure is a type of cellular structures, and it has inherent advantages over foams in terms of mechanical property by the volumetric density of the material. There are randomized, periodic, and pseudoperiodic lattice structures, and the cells of a lattice structure can be homogenous or heterogeneous [161]. Dong et al. [162] reduced the simulation cost for solid-lattice hybrid structures by using beam elements for the lattice and tetrahedral elements for the solid. To connect the two elements that have 
different degrees of freedom, they calibrated the parameter of rigid body element for the connection. Tang et al. [163] took the manufacturing constraints into account during the design and optimization of lattice structure to ensure its manufacturability. By applying size optimization to update the sizes of struts, it can improve the printing quality and the stiffness of the model without increasing the weight of part. Sigmund et al. [164] introduced a method to design the periodic microstructure of a material to obtain prescribed constitutive properties. They formulated the problem of finding the simplest possible microstructure to achieve desired elastic properties as an optimization problem named inverse homogenization problem. Bickel et al. [165] presented a method to measure deformations of base material's structure and stack them to reach a target behavior through combinatorial optimization. Schumacher et al. [166] extended this idea by designing microstructure to match given homogenous material properties. These microstructures are tiled to create objects with spatially varying elastic properties. On the other hand, Panetta et al. [167] introduced a library of tileable parameterized 3D microstructures to control the elastic material properties of an object. By choosing a space of structures with limited but sufficiently large set of parameters, a small family of structures can be optimized to achieve specific material properties.

There are some hybrid approaches combining several methods to improve accurancy and reduce computational cost. For example, Zhang et al. [154] related the cellular microstructure to the topology optimization results at macroscopic scale (Figure 6(e)). The hexagonal structure is reconstructed by mapping the optimized density to the strut diameter. Zhu et al [168] proposed a two-scale topology optimization that computes an optimal topology in the low-level building blocks using a level set field and maps the results onto the microstructures to generate a high-resolution printable structure. They have demonstrated that the method can optimize the material property spaces on the level of a trillion voxels.

\subsubsection{Shape Descriptors}

The decomposition methods discussed above can simplify the problem, but the simplification also sacrifices certain degree of freedom. Some researches model the material properties by using implicit representations or functions (see Figure 6(f)). Under this scheme, the shapes of various regions are not limited by the predefined topology, and very detailed structures can be generated in a large object without having a full explicit representation. The absence of global organization and periodicity allows the free gradation of density, orientation, and stretch, leading to the controllable orthotropic behaviors. The challenge here is how to define the functions or descriptors. One popular approach is to use surfaces that are similar to the biological membranes studied in natural science. Wang [169] proposed the analytical models of triply periodic minimal surface (TPMS) for the nano design. Yoo [170, 171] combined volumetric distance field and TPMS pore morphology to generate porous scaffold design systematically, which is also used to generate projection images for image-based AM processes directly without the need of slicing the complicated 3D scaffold models [172, 173]. Schroder-Turk et al. [174] used Minkowski tensors to extract physically-relevant spatial structure information to quantitatively characterize and analyze the cellular structures. Wang and Rosen [175] presented a new dual representation to model heterogeneous materials based on a new basis function - surfacelet, which can capture material distributions at multiple scales. Huang et al [176] extended the method to a new multiscale specification environment based on material images and microstructural feature modeling (see Figure 6(g)). With both the forward and inverse surfacelet transformation [156], their method has the capability to zoom-in and zoom-out seamlessly to exchange material information at multiple scales.

Localization (as opposed to homogenization) describes the spatial distribution of the response field of interest (stress or strain fields) at the microscale for an imposed loading condition at the macroscale. Fast and Kalidindi [177] applied the Materials Knowledge Systems (MKS), which facilitates bi-directional exchange of information between different scales and calibrates the higher-order terms in the localization relationship to improve the accuracy of multiscale modeling and simulation. Baniassadi et al. [178] applied 
Monte Carlo sampling method to reconstruct microstructure using two-point correlation functions. They have also used on the colony and kinetic algorithms to simulate the virtual microstructures. The lower-order functions can be used to approximate solution for the higher-order ones (n-point correlations) for heterogenous materials [179]. Xu et al. [180] derived 3D descriptors from the 2D images based on the characterization of 2D microstructural morphology. Their method can reconstruct large size 3D structures even when the direct 3D microstructure analysis is not available. Gupta et al. [181] established surrogate models with low computational cost for measuring the microstructures using n-point spatial correlations and principal component analysis (PCA). Huang et al. [182] generalized the periodic surface model to model the shapes of fibers in porous media, and the model can construct the 3D volume element of randomly distributed fibers.

In the multi-scale modeling, the transitions among scales are usually modeled by concurrent and/or hierarchical schemes. However, certain information is lost in either way, and there is no single multi-scale modeling technique sufficient for various applications in different length-scales. Hansmeyer [183] presented a procedural subdivision process to articulate the model at multiple scales, yet the approach is rather limited to certain applications. With the growth of computational power and cloud computing, the concurrent methods will play a very important role - particularly when the response within a microstructure cannot be localized. At the same time, a promising trend of generative design [184] can be applied. Nevertheless, before that, the data-driven methods and dual-representation approaches are the most practical strategy at this moment. At present, the material and manufacturing information of the microstructures can be extracted on-the-fly, so that the demand of memory and commutation is minimized. However, the major challenge is still the high-dimensional optimization during the iterative computation. One possible direction is to convert the optimization problem into a design problem, such that the accuracy does not rely on the resolution of the solution space. But this requires a complete understanding of the problem and the development of design principles for the problem in different scales.

\subsection{DESIGN FOR MULTI-FORM AM PROCESSES}

With the newly tailor-made materials and the capability of multi-material printing technology, we can now fabricate multi-form products using various materials, e.g., plastic, elastomer, composites and shape memory polymer. This offers designers more freedoms to design products with fewer limitations and focus more on the intended forms at different statuses. In this section, we will review the different design methodologies for multi-form fabrication by means of the mechanisms.

\subsubsection{Folding and Origami}

In classical mechanics, motions between rigid bodies are realized by their connections - kinematic pairs or joints. The connection could be located at a point or along a line, such as mechanical linkage or folding. With the help of additive manufacturing, the kinematic pairs can be fabricated directly together with different choices of materials, resulting in a more complex system that can be designed and produced. Wang et al. [185] studied mobility and foldability of foldable mechanisms. Their method is based on the formula of the revolute-joint design related to the joints' degrees of freedom and the order of wrench system. They described that a shape-shifting structure can be folded into the same pattern through different methods and can also be folded to different patterns.

Recently, one of the active topics in folding mechanism is the origami (or also kirigami) designs. Having the ability in transforming back-and-forth between its stowed state and the deployed state (see Figure 7(a)), origami-based mechanisms have many attractive applications, such as the origami wheel presented in [186]. Belcastro and Hull [187] used affine transformations to compute the valid origami structures and the mappings between unfolded and folded configurations. Schenk and Guest [188] proposed 
a model for origami structures with elastic creased folds via truss representations. Their model is based on introducing the behavior of torsional springs at the creases. Tachi [189] used a similar approach to model the elastic behavior of sheets with creased folds by also idealizing the folds as torsional springs. He also solved the equations of mechanical equilibrium under constraints assuring that no fold line or boundary edge is elongated. This model provided the theoretical basis for his origami simulation tools (i.e., the Freeform Rigid Origami Simulator [190]) to compute the unfolded patterns for a given folded target shape. Zhu et al. [191] developed a method for analyzing surfaces under creased and bent folds. Their tool allowed for the superposition of folds with arbitrary sharpness and angle that collectively dictated the ultimate shape of the analyzed surface. Recently, Morgan et al. [192] presented a design process for generating origamiadapted product based on an exemplar-based methodology (see Figure 7(b)). The process includes: (1) evaluating the criteria to verify if the problem is suitable for origami-adapted design, (2) picking seed origami from standard patterns that function close to the need, (3) modifying fold patterns to meet the design requirements, and (4) integrating the pattern with material and prototyping. On the other aspect, modeling a freeform surface using foldable mechanisms is also relevant to the geometric modeling of developable surfaces $[193,194,195]$. In these approaches, an inverse design problem - finding the planar structure for a given $3 \mathrm{D}$ target -
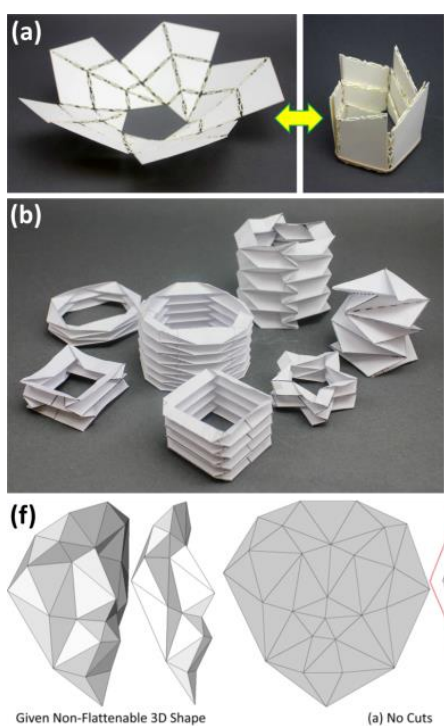
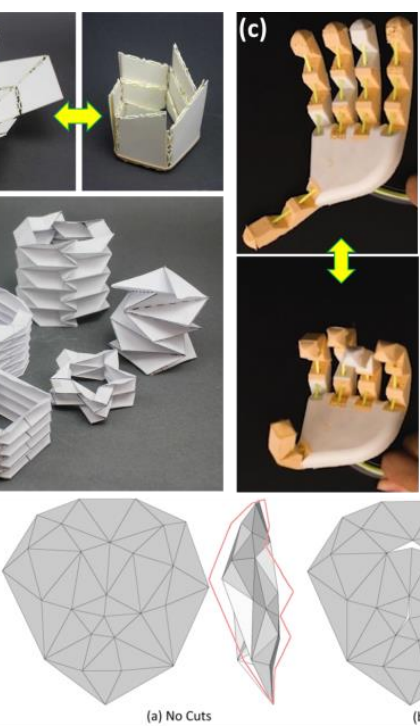

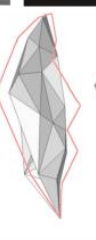

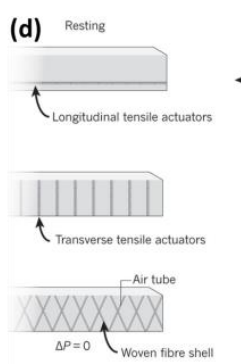

(d) Resting
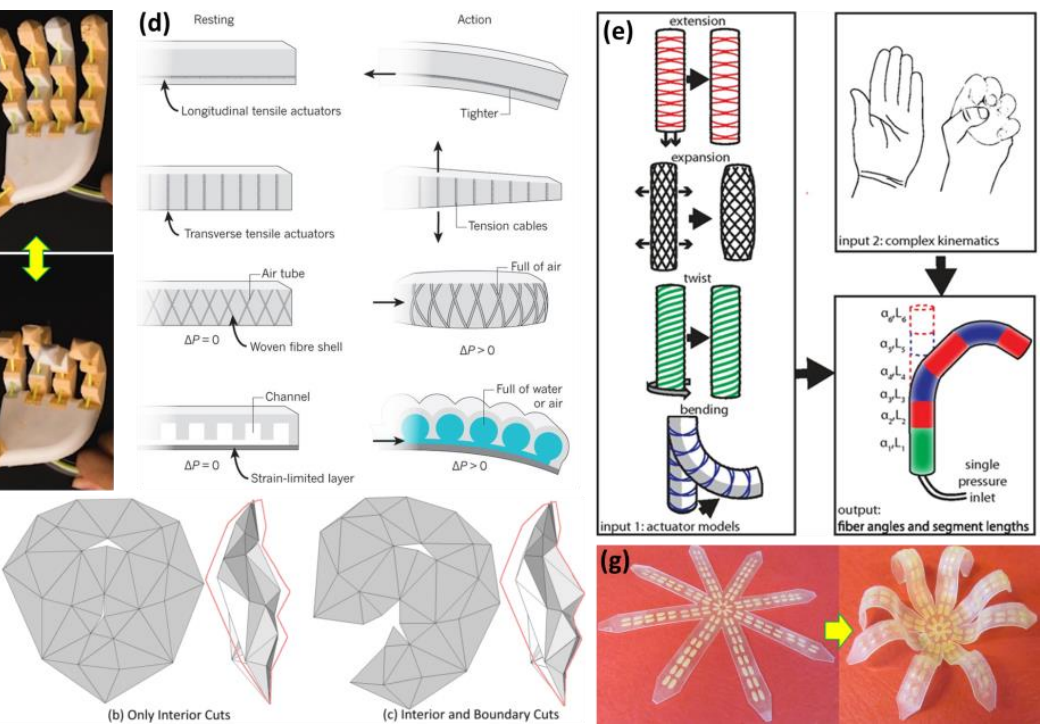

Figure 7 (a) An origami antenna in both stowed and deployed states. (b) The database of origami models that can be applied to a bellows [192]. (c) A hand design using elastomer and joints to achieve different curvatures [196]. (d) Various actuation designs for soft-robots [197]. (e) The database of fiber-reinforced soft actuators are used to design for matching the input trajectory [198]. (f) Shape optimization and cutting are done for 4D-printing non-flattenable object [61]. $(\mathrm{g})$ A self-transformed flower with designed pattern to constrain the shrinkage of a pre-strained film [60].

\subsubsection{Elasticity and Soft Robotics}

With the capability of printing elastomeric and/or rigid materials, we can design certain mechanical deformation to mimic physical objects (see Figure 7(c)) like gripper [196] and heart [199]. Most current practices are computed in a trial-and-error manner, and they design different actuations using tensile cables, constraint fibers, pneumatic bellows, etc. [197] (see Figure 7(d)) and then test the responses and the deformations. Connolly et al. [198] created a database of fiber-reinforced soft actuators, and they developed an automatic selection of mechanical properties to match the kinematic trajectory of a gripper using the exemplars in the database (see Figure 7(e)). Other methods estimate material parameters of constitutive model by considering Young's modulus [200, 201] and acquire complex heterogeneous deformation through optimizing material distribution of Young's modulus [202]. However, the results generated by these approaches may not be manufactured due to the limited materials that can be printed with current AM 
processes. A halfway approach is to design microstructures with single material to approximate elasticity for 3D printing [166, 203, 167], which has been discussed in section 3.2.1.

It is difficult to design directly for performance and manufacturability, but it can be made possible by integrating simulation into the design phase, which allows an iterative process to update the design and estimate the physical effects of the changes. Skouras et al [204] presented a design method for deformable character with a set of target-poses as input. The method combines finite-element analysis (FEA), sparse regularization and constrained optimization in the design phase to compute a set of actuators along with their locations and material distribution such that the resulting character exhibits the desired deformation behavior. As FEA is often the bottleneck in computation, some approaches make use of numerical coarsening $[205,206]$ to speed up the FEA without a huge penalty in accuracy. Another speedup technique is data-driven methods, which is very effective in those applications with interactive response demand [203].

In fact, this idea is common in the fabrication of soft robot, where 3D printing is getting more applied to. While commercial software like Abaqus and COMSOL can be used in soft robot design [207], Hiller and Lipson [208] developed a platform called Voxelyze that is able to generate results of dynamic simulation for multi-material soft objects to get a fast simulation. Voxel representation is used for simulating large deformation and evolutionary computation is employed to obtain optimized material distributions [209]. Nevertheless, many voxels are needed to represent models with complex shape, which will tremendously slow down the computation. SOFA [210] is a widely used framework in the field of surgical and biomedical simulation. Based on SOFA, Duriez et al. [211, 212, 213] developed an asynchronous simulation framework by the trade-off between deformation accuracy and computational speed, such that a real-time simulation of soft robots is realized to support interactive deformation. Their algorithm uses the iterative method to solve ordinary differential equations meanwhile transferring the boundary conditions using Lagrangian multipliers. This method is fast but suffers from the problem of numerical accuracy, particularly if there is large rotational deformation. Wang et al. [214] presented an optimization-based method to find an accurate mathematical simulation model for pneumatic driven grippers. With the help of mass-spring system, Stanley et al. [215] presented a close-loop control for haptic jamming deformable surface. However, they are application-specific and may not be generalized to other domains. Recently, Fang et al. [196] presented a geometry-based approach that can simulate multi-material actuators in large rotational deformation in a quite accurate way.

\subsubsection{Self-Transformation}

While the previous subsections discuss the mechanisms that transform external forces and movement into deformation either by the designed connections or the elastic materials. There is research that focuses on the development of self-folding, self-assembly, or programmable materials to mimic the biological process like DNA [216] for fabricating smarter structures and better products. Many prototypes have been developed with different materials, fabrication technologies, and external energy sources [217]. However, many of these prototypes require an additional production step to embed the 'programmability' and the potential energy for transformation, e.g., adding magnets and elastic strands. This leads to the development of four-dimensional (4D) printing, which streamlines the process of production for programmable and adaptive materials. There are survey papers $[62,64,218]$ reviewing the current 4D printing processes, concepts, and related tools. They have described the different shape-transformation behaviors such as bending and folding, the commonly used materials and structures, and the shape-shifting mechanisms. It has been also mentioned that the design for shape-transformation behavior is needed to predict and model the structures, such that the number of trial-and-error experiments can be reduced.

Gladman et al. [72] studied a bilayer structure that is fabricated by depositing the ink of hydrogel composite in different print paths for the two layers. They defined the elastic and swelling anisotropies by controlling the orientation of cellulose fibrils within the hydrogel, and the orientation is computed by the 
mathematical relationships of desired curvatures and the elastic moduli, the swelling ratios and the layer thicknesses. Kwok et al. [61] pointed out that only developable surfaces can be shaped-transformed from 2D to 3D. Based on differential geometry, they developed a geometry optimization method for freeform surfaces to modify a non-flattenable surface into a flattenable one. The shape optimization framework also supports topological operators to add cuts to improve the flattenability (see Figure 7(f)). Deng et al. [60] used an exemplar-based approach to approximate a smooth surface. They created a library of constraint patterns with controllable variables and studied the relationship between the patterns and the deformation (see Figure 7(g)). A given 3D smoothly curved surface is subdivided into patches and approximated by the patterns in the library. Their method can approximate different folding types, folding orientation, folding axis and folding curvatures. Kwok and Chen [219] developed a Geometry-Driven Finite Element (GDFE) method to simulate shape-transformation behavior efficiently, which can be integrated in the design phrase for an iterative design algorithm. They also demonstrated that by understanding the material distribution and the resultant curvature using the GDFE framework, a mathematical model can be formulated to design for freeform shapes.

In fact, many causes of motion are based on geometric variations such as displacement, rather than by well-defined structural loads. A more direct way in simulating motion by geometric inputs is needed to achieve better convergence and accuracy. A most recent effort can be found in [196]. Furthermore, the simulation solves a forward problem that determines the final shape with given material structures and deformation principles. A more challenging question is how to design the material structure that can be transformed into a desired shape. This is an inverse problem, which is the determination of the material distribution in a structure based on the final desired shape and the deformation principle. The inverse problem is far more complex in determining a discrete small-scale material distribution that yields the desired self-transformation behavior. One direction in solving this problem is to apply pattern learning and synthesis techniques with the data-driven approaches. The 1D-to-2D, 2D-to-3D, and 3D-to-3D shapetransformation behaviors should be studied, and the deformation principles need to be fully understood to design the evolution of 3D structures

\subsection{DESIGN FOR MULTI-FUNCTIONAL AM PROCESSES}

Earlier additive manufacturing techniques are mainly used to realize structural and mechanical properties. But with the recent advance printing technologies [220], AM can be leveraged for use in applications with additional functionalities. Complex multi-functional components can be created, through multi-material and multi-scale printing capabilities, which cannot be realized by conventional fabrication methods. Next, we will provide an overview of design for three popular functional properties.

\subsubsection{Design for Optical Properties}

Advancements in 3D printing technology have led to the ability to create complex optical components, such as microlenses, freeform optics and multi-component optical systems. The unrestricted design freedom and the flexibility to write on arbitrary substrates revolutionized the field of optics. Gissibl et al. [48] presented using 3D direct laser writing for fabricating an optical system with multi-lens that shows high optical performances and tremendous compactness. The system can directly be fabricated on the optical fibre for endoscopic applications. Thiele et al. [37] demonstrated 3D printing of four-lens systems onto a chip to form a multi-aperture camera. Benefit from the printed aspherical free-form surfaces in the design of lens, a future device can become smaller but transfer images with higher resolution. To design such compound lenses, an optical design software is used at first. Then, the optical properties are optimized through simulation, and the performance and functionality are finally validated with fabrication. 
Regarding optical properties, freeform surface allows higher degrees of freedom in optical design, and provides substantially improved imaging performance. In particular, the representation technique of a freeform surface has been a key research topic in recent years since it has a close relationship with design, manufacturing, testing and final application, it encompasses all the processes used in freeform optics [221]. A good representation of freeform surface has the advantages of improving the imaging performance, reducing the number and weight, and further increasing the compactness of optical components. From the manufacturing point of view, it is also important to consider surface quality during the design stage [222]. The surface profile can strongly affect the optical properties. Although post-processing offers significant surface improvements, designing an optional lens with complex geometry must take into account for staircase effect and supporting structure.

\subsubsection{Design for Thermal Properties}

Maintaining acceptable temperature ranges can require complicated techniques and components, depending on the application. Additive manufacturing offers a unique solution for thermal management. The design freedom is the major advantage from additive manufacturing. NASA [223] recently developed a space folding fabric that have four essential functions: reflectivity, passive heat management, foldability and tensile strength. By depositing material in layers, they can add multiple functions to the design. Drycooling channel is another heat management example taking advantage of AM technologies. Felber et al. [224] designed a heat exchanger with a tunable microstructure to enhance heat transfer. They also suggested embedding conductive fillers such as graphite, carbon black or carbon fibers to further improve the conductivity. Other examples include printing molds with designed conformal cooling channel to reduce the cooling time as well as the printing cost [225]. More complicated design for conformal cooling in injection molding can be found in [226], which is further improved to have faster coolant velocity in a spiral cooling channel [227]. Zhang et al. [228] introduced a method for designing personalized orthopedic casts, which are aware of thermal-comfort while satisfying mechanical requirements. They created a hollowed Voronoi tessellation pattern with optimized density based on thermal image to maximize both thermal comfort and mechanical stiffness. Similarly, some researches use lattice structure with defined density distribution for thermal problem. Cheng et al. [229] presented a method to concurrently optimize the density distribution of the lattice infill and the layout of functional features. Asymptotic homogenization is employed to obtain the effective thermal conductivity of lattice structure in terms of the relative density.

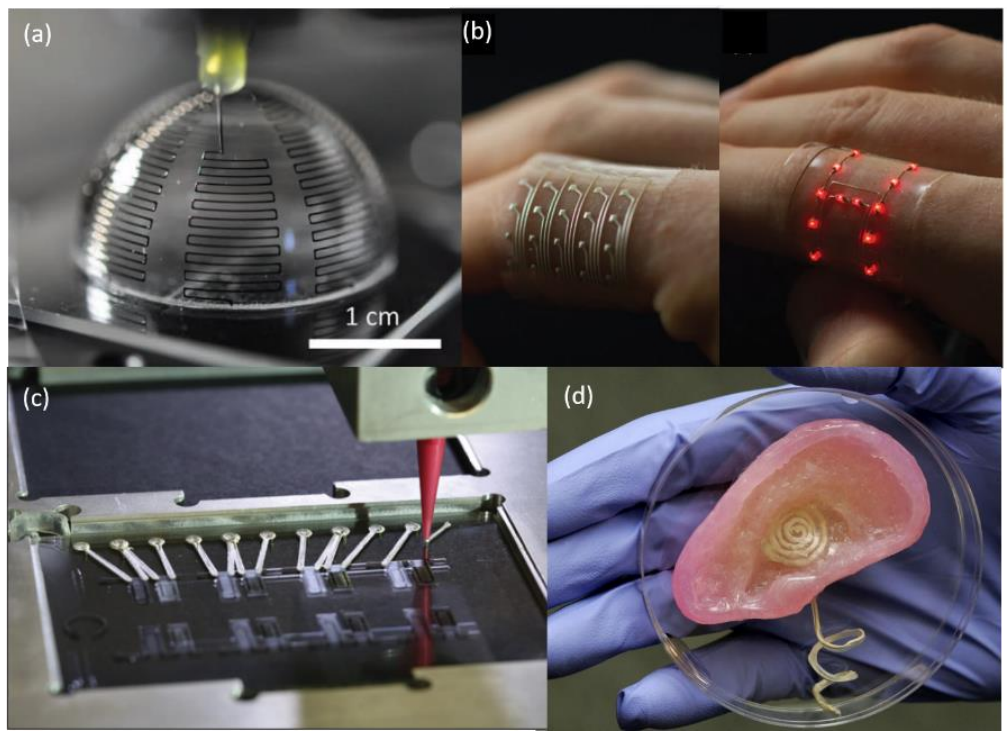

Figure 8 (a) An electrically small antenna [230]. (b) A stretchable device with LED arrays mounted on it [93]. (c) Heart-on-thechips with integrated sensing [231]. (d) A bionic ear that can hear radio frequencies a million time higher than human ears [232]. 


\subsubsection{Design for Electrical Properties}

With right kind of 3D printer, it is also possible to build parts with embedded electronic. The process integrates substrates, conductive inks and electronic components within the 3D-printed objects, allowing a degree of design freedom that is simply not possible through standard methods of manufacturing. For example, Adams et al. [230] demonstrated how to fabricate a 3D electrically small antenna by conformally printing metallic inks onto convex and concave hemispherical surface (Figure 8(a)). The 3D design enabled the specification of both operating frequency and size, achieving near-optimal bandwidth at several frequencies of interest wireless communications. The ability to conformally print antennas that are compact and encapsulated within their support substrate further enhanced the mechanical robustness. Not only special type of surface, Valentine et al. [93] printed soft stretchable electronics that combines direct ink writing with automated pick-and-place of surface mount electrical components (Figure 8(b)). Their hybrid method enabled surface mount electrical components of arbitrary shapes and sizes onto printed soft wearable circuits, offering a unique opportunity to expand the functionality of 3D printed structures. With the capability to print on arbitrary surface, it is necessary to consider how to perform 3D placement and routing in a functional way. Panesar et al. [233] introduced a design framework to optimize a multifunctional 3D part by coupling both the functional system and the structural design aspects. They proposed methods to select locations of components based on a performance and/or geometry criterion, and identify component orientation using the skeletal information. They also suggested to use accurate or approximate shortest path computation approaches to generate connection and form a circuit, i.e. routing. Optimizing routing can improve the circuit efficiency by lowering resistance, which is proportional to the conductive track length. Later, they used coupling strategies to solve (1) the placement and routing problems with heuristic approach, and (2) the structural optimization with the placement and routing via topology optimization algorithms in [234].

Printing electronic components seamlessly with biological tissue and organs is even more complicated. Lind et al. [231] utilized multi-material 3D printing technology to print six materials at microscale, which enabled the integration of soft strain gauge sensors in cardiac micro-physiological devices (Figure 8(c)). In [232], a bionic ear was printed to demonstrate the feasibility of enhancing human functionalities (Figure $\mathbf{8}(\mathrm{d})$ ). For biological applications, the printed devices are required to match geometries, mechanical and biochemical properties to every cell source/material, making the design process ever complicated. Ultimately, like Festo [235] who has taken the delicate anatomy of the natural ant as a role model and control bionic ants to complete complex task together, they brought the integration of AM technologies into another level.

Multifunctional AM technology has become an intensive research field. Researchers combine the multi-material and multi-scale printing capabilities to create different multifunctional devices for diverse applications. Meanwhile, there is no automatic way to categorize the information with respect to the functionalities and provide effective insight for different designs. Researchers usually need to equip with knowledge in various divisions of field to interpret the problem. Insufficient understanding could lead to design cycles with long time and difficulty in obtaining an optimal design. How to integrate the information from the individual functions in different aspects would be the key factor for a sustainable development.

\section{CONCLUSION AND OUTLOOK}

Additive manufacturing is a very promising manufacturing process in terms of the geometry complexity and functionality. Because of the advanced development of AM processes, and the diversity of programmed materials appeared, we can summarize a framework (as shown in Figure 9) to design functional part with feasible processes and materials. Besides, we also introduce the work that has get insufficient attention and discuss some possible directions for future research. 


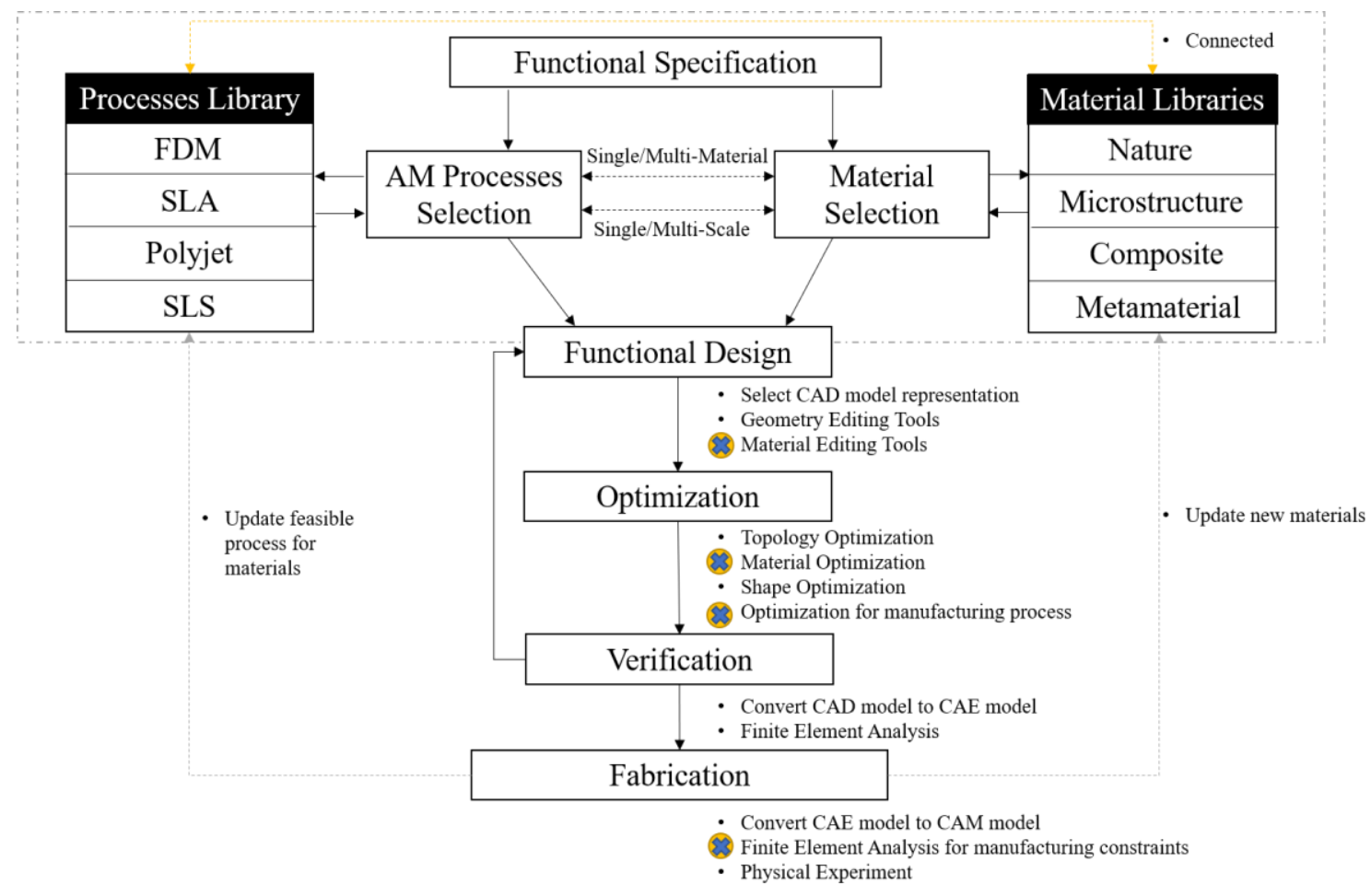

Figure 9 Design framework for functional additive manufacturing. Crossed bullet points suggest the directions are getting insufficient attention.

(1) Based on the given functional requirements, our design framework begins with the selection of AM processes and material selection. AM process has shown great advantages in fabricating multifunctional structures. But there are still several problems needs to be solved. For example, the multifunctionality usually needs multi-material 3D printing process, and the kinds of materials needs to be broadened. The study to broaden the kinds of materials that can be 3D printed as well as to develop novel composites is a critical task in the future. Besides, it is very challenging for current 3D printing technology to process materials in different categories due to the incompatible forming conditions. New material deposition mechanisms may also enable multi-material 3D printing with higher resolution and faster speed. In the hydrodynamic level, some multifunctional bioinspired structures possess multiscale features, which are hard for the 3D printing process to replicate. The further development of multifunctional AM process will lie in the combination of multi-material and multi-scale AM technology. A database linking AM processes, material and functional specification becomes emergently needed.

As shown in Figure 9, we establish two libraries which allow the interoperability (yellow dotted line) between them. A comprehensive collection of materials (includes programmed materials) with experimental data from fabrication processes supports designers to determine how the functions relate to the manufacture constraints and material properties. As such, every profile of materials should be linked with at least one process, and each process should provide a list of manufacturable materials along with the necessary parameters. The connection guarantees that any profiles created in the libraries are feasible and practicable. In the AM process/Material selection stage, there exists a two-way coupling relationship (as shown in black dotted arrows). The relationship indicates that not only the process could be affected by the available materials, the material selection is also affected by the manufacturability. Therefore, in the early stage of functional design, it is very important to be guided by this information. 
(2) One of the important challenges is to set an appropriate representation model that are reliable, reasonably accurate, computationally efficient, user-friendly, and easy to modify, share and store [236]. For example, the overall characterization of microstructures needs to be improved. A standard protocol for assessing the dimensions/microstructure/mechanical performance needs to be developed. Multifunctional additive manufacturing is a new emerging technology and still being under development. As this area of manufacturing is nascent, most ongoing research go into materials and process configuration. Little works have been carried out on developing the design methodologies that specifically for multi-functional AM processes. Designing a part with pre-fabricated components (e.g. circuits, sensors, etc.) requires a more complex design representation when compared to a simple mechanical structure, due to the existence of non-mechanical features, e.g. optical, electronic features. These simultaneous features require more than one $\mathrm{CAD}$ methodologies, and their combination/coupling issues are manually handled in current research. A thorough designconsideration for the currently existing procedures and applications are needed. In the future, it is expected to have proper operations and representation to allow design on arbitrary geometries, arbitrary material composition, and arbitrary object scales.

(3) From the review, it is found that most of the current design methodology is through an iterative process to optimize the design-based simulation results. The common tool in computer-aided engineering (CAE) for estimating the behavior of an object is FEM. However, the framework of FEM is known to be computational expensive, and relies on the accurate input of structural loads and material properties. As a consequence, simplifying the FEM computation is an active and emerging topic, especially when the capability of AM is extending to multiple materials with complex shape and topology. When the functional specifications are getting more complicated, efficient and effective optimization methods on geometry and materials are particularly essential at present.

(4) Eliminating the gap between the design and the manufacturing processes is also very important. We need to ensure that the finally fabricated product reflects the design as best as possible. One major factor that lead to discrepancy is the human involvement during the design-to-fabrication process. Automating some functions, such as process parameter controls (e.g. extrusion temperature, overlap percentage) and design constraints (e.g. building orientation, material selection) could significantly reduce mistakes made during the operation. In other words, managing and interpreting data has come to be regarded as a key concern across design-to-manufacturing process. In particular, when it comes to multiple materials, which makes the process hard to control - data is certainly needed for reproduction. To establish the foundation for future AM industry, one way is to associate the data with the mentioned library, which consists of comprehensive description for each digital material. The library is then opened for assessment in different applications. Therefore, whenever a new digital material is successfully printed, the database will be updated for verification. In long-term, using machine to "read" the library and assist in design stage could handle complex problems much better.

(5) From the process perspective, despite the recent advances in 3D printing, challenges that need to be addressed in future still exist. For example, the material selection for the 3D printing of shape-changing structures is still limited. Mainly a few categories of polymers are currently available, which significantly restricts the potential applications of shape-changing structures. Moreover, the stimuli used to trigger shape-changing movements are still based on laboratory environments, and might not be broadly applied in the real world. Meanwhile, the shape-changing behaviors triggered by these stimuli have big variations and cannot be reliably are accurately controlled, which makes it difficult to be applied in an actual human-centered system. In the future, new materials need to be explored and 
incorporated into structures to achieve multifunctional properties - such as ceramics with mechanical rigidity, scaffolds with bio-degradability, chemical or thermal resistance, etc.

In addition, multi-material processing in micro- and nano- scales using 3D printing technology is difficult to achieve due to the resolution limitation. The material blur, existing in adjacent regions of different 3D printed materials, is one of the crucial problems restricting the resolution of multi-material printing technology. As we discussed, an effective material changing system is necessary for multimaterial printing process to achieve desired functional performance on the printed structures. Regardless, researchers have made strides to expand the dimensional span of 3D printing technologies, achieving fabricated feature sizes ranging from dozens of millimeters to several microns. Recent progress on the development of multi-scale 3D printing technologies also shows applications of 3D printing as a tool to validate the design with advanced material and the system with multi-scale structures. For known limitations of 3D printing process, including the printing efficiency, the printing material, and the printing complexity, recent researches also reveal a feasible solution that is to leverage traditional manufacturing process through synthesizing and integration. Such integration of multiple printing processes and traditional fabrication processes provides the capability to build multi-scale and multi-material functional objects, and is still a trending topic in upcoming future.

At present, many research groups are exploiting the fabrication capabilities to meet the everchanging needs for high-performance functions. It has led to the ongoing development of new design methodologies for integrating the state-of-the-art AM technologies and customized programmable materials, which enables the never-before possible performance or products exist. In the future, we envision a more collaborative and connective in between the design and the manufacturing communities, together with interdisciplinary teams to address complex design challenges and accomplish the next generation of computational design-to-manufacturing system.

\section{ACKNOWLEDGMENTS}

The authors acknowledge the support from National Science Foundation (NSF) (Grant Nos. CMMI 1151191 and CMMI 1663663) and USC's Daniel J. Epstein Institute.

\section{REFERENCES}

[1] Altair, [Online]. Available: https://altairhyperworks.com/product/OptiStruct.

[2] Z. Hu, K. Thiyagarajan, A. Bhusal, T. Letcher, Q. Fan, Q. Liu and D. Salem, "Design of ultralightweight and high-strength cellular structural composites inspired by biomimetics," Composites Part B: Engineering, vol. 121, pp. 108-121, 2017.

[3] B. Compton and J. Lewis, "3D-Printing of Lightweight Cellular Composites," Advanced Materials, vol. 26, no. 34, 2014.

[4] G. X. Gu, M. Takaffoli, A. Hsieh and M. Buehler, "Hierarchically enhanced impact resistance of bioinspired composites," Advanced Materials , vol. 29, no. 28, p. 1700060, 2017.

[5] Y. Yang, Z. Chen, X. Song, Z. Zhang, J. Zhang, K. K. Shung, Q. Zhou and Y. Chen, "Biomimetic Anisotropic Reinforcement Architectures by Electrically Assisted Nanocomposite 3D Printing," Advanced Materials, vol. 29, no. 11, 2017.

[6] K. Wang, C. Wu, Z. Qian, C. Zanga and B. Wanga, "Dual-material 3D printed metamaterials with tunable mechanical properties for patient-specific tissue-mimicking phantoms," Additive Manufacturing, vol. 12, pp. 31-37, 2016. 
[7] K. K. Saxena, E. P. Calius and R. Das, "Tailoring cellular auxetics for wearable applications with multimaterial 3D printing," in ASME 2016 International Mechanical Engineering Congress and Exposition, Phoenix, Arizona, USA, 2016.

[8] I. M. El-Sherbiny and M. H. Yacoub, "Hydrogel scaffolds for tissue engineering: progress and challenges," Global Cardiology Science \& Practice, vol. 3, pp. 316-342, 2013.

[9] Y. Yang, X. Li, X. Zheng, Z. Chen, Q. Zhou and Y. Chen, "3D-Printed Biomimetic SuperHydrophobic Structure for Microdroplet Manipulation and Oil/Water Separation," Advanced Materials, vol. 30, no. 9, 2018.

[10] M. Vaezi, H. Seitz. and S. Yang, "A review on 3D micro-additive manufacturing technologies," The International Journal of Advanced Manufacturing Technology, vol. 67, no. 5-8, pp. 1721-1754, 2013.

[11] W. E. Frazier, "Metal additive manufacturing: a review," Journal of Materials Engineering and Performance, vol. 23, no. 6, pp. 1917-1928, 2014.

[12] S. Huang, P. Liu, A. Mokasdar and L. Hou, "Additive manufacturing and its societal impact: a literature review," The International Journal of Advanced Manufacturing Technology, vol. 67, no. 5-8, pp. 1191-1203, 2013.

[13] W. Gao, Y. Zhang, D. Ramanujan, K. Ramani, Y. Chen, C. B. Williams, C. C. Wang, Y. C. Shin, S. Zhang and P. D. Zavattieri, "The Status, Challenges, and Future of Additive Manufacturing in Engineering," Computer Aided Design, pp. 65-89, 2015.

[14] N. Guo and M. Leu, "Additive manufacturing: technology, applications and research needs," Frontiers of Mechanical Engineering, vol. 8, no. 3, pp. 215-243, 2013.

[15] Y. L. Kong, I. A. Tamargo, H. Kim, B. N. Johnson, M. K. Gupta, T.-W. Koh, H.-A. Chin, D. A. Steingart, B. P. Rand and M. C. McAlpine, "3D printed quantum dot light-emitting diodes," Nano letters, vol. 14, no. 12, p. 7017-7023, 2014.

[16] W. Liu, Y. S. Zhang, M. A. Heinrich, F. D. Ferrari, H. L. Jang, S. M. Bakht, M. M. Alvarez, J. Yang, Y.-C. Li, G. T.-d. Santiago, A. K. Miri, K. Zhu, P. Khoshakhlagh, G. Prakash, H. Cheng, X. Guan, Z. Zhong, J. Ju, G. H. Zhu, X. Jin and S. R. Shin, "Rapid continuous multimaterial extrusion bioprinting," Advanced Materials, vol. 29, no. 3, 2016.

[17] Q. Wang, J. Jackson, Q. Ge, J. Hopkins, C. Spadaccini and N. Fang, "Lightweight mechanical metamaterials with tunable negative thermal expansion," Physical review letters, vol. 117, no. 17, p. 175901, 2016.

[18] D. Espalin, J. A. Ramirez, F. Medina and R. Wicker, "Multi-material, multi-technology FDM: exploring build process variations," Rapid Prototyping Journal, vol. 20, no. 3, pp. 236-244, 2014.

[19] N. Oxman., E. Tsai and M. Firstenberg, "Digital anisotropy: A variable elasticity rapid prototyping platform," Virtual and Physical Prototyping, vol. 7, no. 4, pp. 261-274, 2012.

[20] P. Sitthi-Amorn, J. E. Ramos, Y. Wangy, J. Kwan, J. Lan, W.Wang and W. Matusik, "MultiFab: a machine vision assisted platform for multi-material 3D printing," ACM Transactions on Graphics, vol. 34, no. 4, 2015.

[21] H. Yang, J. Lim, Y. Liu, X. Qi, Y. Yap, V. Dikshit, Y. W.Y and J. Wei, "Performance evaluation of ProJet multi-material jetting 3D printer," Virtual and Physical Prototyping , vol. 12, no. 1, pp. 95103, 2017. 
[22] K. Willis, E. Brockmeyer, S. Hudson and I. Poupyrev, "Printed Optics: 3D Printing of Embedded Optical Elements for Interactive Devices," in Proceedings of the 25th annual ACM symposium on User interface software and technology, Cambridge, Massachusetts, USA, 2012.

[23] X. Li and Y. Chen, "Micro-scale feature fabrication using immersed surface accumulation," Journal of Manufacturing Processes, vol. 28, no. 3, pp. 531-540, 2017.

[24] Q. Ge, A. Sakhaei, H. Lee, C. Dunn, N. Fang and M. Dunn, "Multimaterial 4D printing with tailorable shape memory polymers," Scientific report 6, p. 31110, 2016.

[25] J.-W. Choi, H.-C. Kim and R. Wicker, "Multi-material stereolithography," Journal of Materials Processing Technology, vol. 211, no. 3, pp. 318-328, 2011.

[26] C. Zhou, Y. Chen, Z. Yang and B. Khoshnevis, "Development of multi-material mask-imageprojection-based stereolithography for the fabrication of digital materials," in International symposium; 22nd, Solid freeform fabrication, Austin, TX, 2011.

[27] R. Melnikova, A. Ehrmann and K. Finsterbusch, "3D printing of textile-based structures by Fused Deposition Modelling (FDM) with different polymer materials," IOP Conference Series: Materials Science and Engineering, vol. 62, no. 1, 2014.

[28] M. Jafari, W. Han, F. Mohammadi, A. Safari, S. Danforth and N. Langrana, "A novel system for fused deposition of advanced multiple ceramics," Rapid Prototyping Journal, vol. 6, no. 3, pp. 161$175,2000$.

[29] A. Salea, R. Prathumwan, J. Junpha and K. Subannajui, "Metal oxide semiconductor 3D printing: preparation of copper (ii) oxide by fused deposition modelling for multi-functional semiconducting applications," Journal of Materials Chemistry C, vol. 5, no. 19, pp. 4614-4620, 2017.

[30] M. Vaezi, S. Chianrabutra, B. Mellor and S. Yang, "Multiple material additive manufacturing - Part 1: a review," Virtual and Physical Prototyping, vol. 8, no. 1, pp. 19-50, 2013.

[31] W. Wu, A. DeConinck and J. Lewis, "Omnidirectional printing of 3D microvascular networks," Advanced Materials, vol. 23, no. 24, 2011.

[32] Z. Qin, B. Compton, J. Lewis and M. Buehler, "Structural optimization of 3D-printed synthetic spider webs for high strength," Nature Communications volume 6, 2015.

[33] L. Wen, J. Weaver and G. Lauder, "Biomimetic shark skin: design, fabrication and hydrodynamic function.," Jounral of Experimental Biology, vol. 217, pp. 1656-1666, 2017.

[34] L. Bai, Z. Xie, W. Wang, C. Yuan, Y. Zhao, Z. Mu, Q. Zhong and Z. Gu, "Bio-inspired vaporresponsive colloidal photonic crystal patterns by inkjet printing," ACS Nano, vol. 8, no. 11, p. 11094-11100, 2014.

[35] M. Lee, G. Cooper, T. Hinkley, G. Gibson, M. Padgett and L. Cronin, "Development of a 3D printer using scanning projection stereolithography," Scientific Reports, vol. 5, 2015.

[36] H. Mao, Y.-S. Leung, Y. Li, P. Hu, W. Wu and Y. Chen, "Multiscale Stereolithography Using Shaped Beams," Journal of Micro and Nano-Manufacturing, vol. 5, no. 4, 2017.

[37] S. Thiele, K. Arzenbacher, T. Gissibl, H. Giessen and A. Herkommer, "3D-printed eagle eye: Compound microlens system for foveated imaging," Science Advances, vol. 3, no. 2, 2017.

[38] K. Liu and J. Lei, "Multifunctional integration: from biological to bio-inspired materials," ACS Nano, vol. 5, no. 9, p. pp 6786-6790, 2011.

[39] J. Choi, M. Yamashita, J. Sakakibara, Y. Kaji, T. Oshika and R. Wicker, "Combined micro and macro additive manufacturing of a swirling flow coaxial phacoemulsifier sleeve with internal microvanes," Biomed Microdevices, vol. 12, no. 5, pp. 875-886, 2010. 
[40] X. Li, T. Baldacchin, X. Song and Y. Chen, "Multi-scale additive manufacturing: an investigation on building objects with macro-, micro- and nano-scales features," in The 11th International Conference on Micro Manufacturing, CA, 2016.

[41] X. Li, Y. Yang and Y. Chen, "Bio-inspired Micro-Scale Texture Fabrication based on Immersed Surface Accumulation Process," in Proceedings of the World Congress on Micro and Nano Manufacturing Conference, 2017.

[42] Y. Chen, H. Mao and X. Li, "Mask Video Projection Based Stereolithography with Continuous Resin Flow". University of Southern California Patent US20160368210A1, 2016.

[43] Y. Pan, X. Zhao, C. Zhou and Y. Chen, "Smooth surface fabrication in mask projection based stereolithography," Journal of Manufacturing Processes, vol. 14, no. 4, pp. 460-470, 2012.

[44] M. Emami, F. Barazandeh and F. Yaghmai, "Scanning-projection based stereolithography: Method and structure," Sensors and Actuators A: Physical, vol. 218, no. 1, pp. 116-124, 2014.

[45] Y.-M. Ha, I.-B. Park, H.-C. Kim and S.-H. Le, "Three-dimensional microstructure using partitioned cross-sections in projection microstereolithography," International Journal of Precision Engineering and Manufacturing, vol. 11, no. 2, p. 335-340, 2010.

[46] Y. Pan and C. Dagli, "Dynamic Resolution Control in a Laser Projection based Stereolithography System," Rapid Prototyping Journal, vol. 23, no. 1, 2016.

[47] Y. Li, H. Mao, H. Liu, Y. Yao, Y. Wang, B. Song, Y. Chen and W. Wu, "Stereolithography with variable resolutions using optical filter with high-contrast gratings," Journal of Vacuum Science \& Technology B, vol. 33, no. 6, 2015.

[48] T. Gissibl, S. Thiele, A. Herkommer and H. Giessen, "Two-photon direct laser writing of ultracompact multi-lens objectives," Nature Photonics, vol. 10, p. 554-560, 2016.

[49] D. Kolesky, R. Truby, A. Gladman, T. Busbee, K. Homan and J. Lewis, "3D bioprinting of vascularized, heterogeneous cell-laden tissue constructs," Advanced Materials, vol. 26, no. 19, pp. 3124-3130, 2014.

[50] A. Au, W. Huynh, L. Horowitz and A. Folch, "3D-printed microfluidics," Angewandte Chemie International Edition, vol. 55, no. 12, pp. 3862-3881, 2016.

[51] C. O'Bryan, T. Bhattacharjee, S. Hart, C. Kabb, K. Schulze, I. Chilakala, B. Sumerlin, W. Sawyer and T. Angelini, "Self-assembled micro-organogels for 3D printing silicone structures," Science Advances, vol. 3, no. 5, 2017.

[52] T. Hinton, Q. Jallerat, R. N. Palchesko, J. Park, M. Grodzicki, H.-J. Shue, M. Ramadan, A. Hudson and A. Feinberg, "Three-dimensional printing of complex biological structures by freeform reversible embedding of suspended hydrogels," Science advances, vol. 1, no. 9, 2015.

[53] T. Bhattacharjee, S. Zehnder, K. Rowe, S. Jain, R. Nixon, W. Sawyer and T. Angelini, "Writing in the granular gel medium," Science Advances, vol. 1, no. 8, 2018.

[54] V. Lee, A. Lanzi, H. Ngo, S.-S. Yoo, P. Vincent and G. Dai, "Generation of multi-scale vascular network system within 3D hydrogel using 3D bio-printing technology," Cellular and Molecular Bioengineering, vol. 7, no. 3, 2014.

[55] Y. Yang, X. Song, X. Li, Z. Chen, C. Zhou, Q. Zhou and Y. Chen, "Recent Progress in Biomimetic Additive Manufacturing Technology: From Materials to Functional Structures," Advanced Materials, no. 1706539, 2018.

[56] S. Tibbits, "4D printing: multi-material shape change," Architectural Design, vol. 84, no. 1, pp. 116121, 2014. 
[57] S. Tibbits, C. McKnelly, C. Olguin, D. Dikovsky and S. Hirsch, "4D Printing and universal transformation," in Proceedings of the 34th Annual Conference of the Association for Computer Aided Design in Architecture, Los Angeles, 2014.

[58] Q. Ge, H. Qi and M. Dunn, "Active materials by four-dimension printing," Applied Physics Letters, vol. 103, no. 13, 2013.

[59] D. Deng and Y. Chen, "Origami-based self-folding structure design and fabrication using projection based stereolithography," Journal of Mechanical Design, vol. 137, no. 2, 2015.

[60] D. Deng, T.-H. Kwok and Y. Chen, "Four-Dimensional Printing: Design and Fabrication of Smooth Curved Surface Using Controlled Self-Folding," Journal of Mechanical Design, vol. 139, no. 8, p. $081702,2017$.

[61] T.-H. Kwok, C. C. L. Wang, D. Deng, Y. Zhang and Y. Chen, "Four-Dimensional Printing for Freeform Surfaces: Design Optimization of Origami and Kirigami Structures," Journal of Mechanical Design, vol. 137, no. 11, p. 111413, 2015.

[62] F. Momeni, S. Hassani.N, X. Liu and J. Ni, "A review of 4D printing," Materials \& Design, vol. 122, pp. 42-79, 2017.

[63] Z. Ding, C. Yuan, X. Peng, T. Wang, H. Qi and M. Dunn, "Direct 4D printing via active composite materials," Science Advances, vol. 3, no. 4, 2017.

[64] Z. X. Khoo, J. E. M. Teoh, Y. Liu, C. K. Chua, S. Yang, J. An, K. F. Leong and W. Y. Yeong, "3D printing of smart materials: A review on recent progresses in 4D printing," Virtual and Physical Prototyping, vol. 10, no. 3, pp. 103-122, 2015.

[65] M. Chae, D. Hunter-Smith, I. De-Silva, S. Tham, R. Spychal and W. Rozen, "Four-dimensional (4D) printing: a new evolution in computed tomography-guided stereolithographic modeling. Principles and application," Journal of Reconstructive Microsurgery, vol. 31, no. 6, 2015.

[66] Q. Ge, C. Dunn, H. Qi and M. Dunn, "Active origami by 4D printing," Smart Materials and Structures, vol. 23, no. 9, 2014.

[67] A. Sidorenko, T. Krupenkin, A. Taylor, P. Fratzl and J. Aizenberg, "Reversible switching of hydrogel-actuated nanostructures into complex micropatterns," Science, vol. 315, no. 5811, 2007.

[68] D. Deng, Y. Yang, Y. Chen, X. Lan and J. Tice, "Accurately controlled sequential self-folding structures by polystyrene film," Smart Materials and Structures, vol. 26, no. 8, 2017.

[69] H. Yang, W. Leow, T. Wang, J. Wang, J. Yu, K. He, D. Qi, C. Wan and X. Chen, "3D Printed Photoresponsive Devices Based on Shape Memory Composites," Advanced Materials, vol. 29, no. 33, 2017.

[70] Y. Liu, B. Shaw, M. Dickey and J. Genzer, "Sequential self-folding of polymer sheets," Science Advances, vol. 3, no. 3, 2017.

[71] L. Huang, R. Jiang, J. Wu, J. Song, H. Bai, B. Li, Q. Zhao and T. Xie, "Ultrafast Digital Printing toward 4D Shape Changing Materials," Advanced Materials, vol. 29, no. 7, 2017.

[72] A. Gladman, E. Matsumoto, R. Nuzzo, L. Mahadevan and J. Lewis, "Biomimetic 4D printing," Nature Materials, vol. 15, pp. 413-418, 2016.

[73] S. Armon, E. Efrati, R. Kupferman and E. Sharon, "Geometry and mechanics in the opening of chiral seed pods," Science, vol. 333, no. 6050, 2011.

[74] D. Correa, A. Papadopoulou, C. Guberan, N. Jhaveri, S. Reichert, A. Menges and S. Tibbits, "3DPrinted Wood: Programming Hygroscopic Material Transformations," $3 D$ Printing and Additive Manufacturing, vol. 2, no. 3, 2015. 
[75] F. L. Bargardi, H. L. Ferrand, R. Libanori and A. Studart, "Bio-inspired self-shaping ceramics," Nature Communications, vol. 7, p. 13912, 2016.

[76] R. M. Erb, J. S. Sander, R. Grisch and A. Studart, "Self-shaping composites with programmable bioinspired microstructures," Nature Communications 4, p. 1712, 2013.

[77] D. Kokkinis, M. Schaffner and A. Studart, "Multimaterial magnetically assisted 3D printing of composite materials," Nature Communications, vol. 6, p. 8643, 2015.

[78] Q. Zhang, D. Yan, K. Zhang and G. Hu, "Pattern transformation of heat-shrinkable polymer by threedimensional (3D) printing technique," Scientific Reports, vol. 5, p. 8936, 2015.

[79] J. T. Muth, D. M. Vogt, R. L. Truby, Y. Mengüç and D. B. Kolesky, "Embedded 3D Printing of Strain Sensors within Highly Stretchable Elastomers," Advanced Materials, vol. 26, no. 36, 2014.

[80] K. Chizari, M. Daoud, A. Ravindran and D. Therriault, "3D Printing of Highly Conductive Nanocomposites for the Functional Optimization of Liquid Sensors Small," Small, vol. 12, no. 44, pp. 6076-6082, 2016.

[81] A. Frutiger, J. Muth, D. Vogt, Y. Menguc, A. Campo, A. Valentine, C. Walsh and J. Lewis, "Capacitive soft strain sensors via multicore-shell fiber printing," Advanced Materials, vol. 27, no. 15, pp. 2440-2446, 2015.

[82] K. Kim, J. Park, J.-H. Suh, M. Kim, Y. Jeong and I. Park, "3D printing of multiaxial force sensors using carbon nanotube (CNT)/thermoplastic polyurethane (TPU) filaments," Sensors and Actuators A: Physical, vol. 263, pp. 493-500, 2017.

[83] Z. Lei, Q. Wang and P. Wu, "A multifunctional skin-like sensor based on a 3D printed thermoresponsive hydrogel," Materials Horizons, vol. 4, pp. 694-700, 2017.

[84] Y. Yang, Z. Chen, X. Song, B. Zhu, T. Hsiai, P.-I. Wu, R. Xiong, J. Shi and Y. Chen, "Three dimensional printing of high dielectric capacitor using projection based stereolithography method," Nano Energy, vol. 22, pp. 414-421, 2016.

[85] Z. Chen, X. Song, L. Lei, X. Chen, C. Fei, C. Chiu, X. Qian, T. Ma, Y. Yang and K. Shung, "3D printing of piezoelectric element for energy focusing and ultrasonic sensing," Nano Energy, vol. 27, pp. 78-86, 2016.

[86] J. Lv, Z. Gong, Z. He, J. Yang, Y. Chen, C. Tang, Y. Liu, M. Fan and W.-M. Lau, "3D printing of a mechanically durable superhydrophobic porous membrane for oil-water separation," Journal of Materials Chemistry A, no. 24, 2017.

[87] S.-Z. Guo, K. Qiu, F. Meng, S. Park and M. McApine, "3D Printed Stretchable Tactile Sensors," Advanced Materials, vol. 29, no. 27, 2017.

[88] M. Darabi, A. Khosrozadeh, R. Mbeleck, Y. Liu, Q. Chang, J. Jiang, J. Cai, Q. Wang, G. Luo and M. Xing, "Skin-Inspired Multifunctional Autonomic-Intrinsic Conductive Self-Healing Hydrogels with Pressure Sensitivity, Stretchability, and 3D Printability," Advanced Materials, vol. 29, no. 31, 2017.

[89] X. Liu, H. Yuk, S. Lin, G. Parada, T.-C. Tang, E. Tham, C. Fuente-Nunez, T. Lu and X. Zhao, "3D Printing of Living Responsive Materials and Devices," Advanced Materials, vol. 30, no. 4, 2018.

[90] Y. Yao, K. Fu, C. Yan, J. Dai, Y. Chen, Y. Wang, B. Zhang, E. Hitz and L. Hu, "Three-dimensional printable high-temperature and high-rate heaters," ACS Nano, vol. 10, no. 5, pp. 5272-5279, 2016.

[91] S. Shin, R. Farzad, A. Tamayol, V. Manoharan, P. Mostafalu, Y. Zhang, M. Akbari, S. Jung, D. Kim, M. Comotto, N. Annabi, F. Al-Hazmi, M. Dokmeci and A. Khademhosseini, "Bioactive 
Carbon Nanotube-Based Ink for Printing 2D and 3D Flexible Electronics," Advanced Materials, vol. 28, no. 17, 2016.

[92] A. Jakus, E. Secor, A. Rutz, S. Jordan, M. Hersam and R. Shah, "Three-dimensional printing of high-content graphene scaffolds for electronic and biomedical applications," ACS Nano, vol. 9, no. 4, 2015.

[93] A. D. Valentine, T. Busbee, J. Boley, J. Raney, A. Chortos, A. Kotikian, J. Berrigan and M. Durstock, "Hybrid 3D Printing of Soft Electronics," Advanced Materials, vol. 29, no. 40, 2017.

[94] T. Brochu and R. Schmidt, "Geometric Modeling of Multi-Material Printed Objects," in Eurographics, France, 2017.

[95] O. Al-Ketan, R. K. A. Al-Rub and R. Rowshan, "Mechanical Properties of a New Type of Architected Interpenetrating Phase Composite Materials," Advanced Materials Technologies, vol. 2, no. 2, 2017.

[96] A. Garland and G. Fadel, "Design and Manufacturing Functionally Gradient Material Objects With an Off the Shelf Three-Dimensional Printer: Challenges and Solutions," Journal of Mechanical Design, vol. 137, no. 11, 2015.

[97] Y.-S. Leung, T.-H. Kwok, H. Mao and Y. Chen, "Digital Material Design for Additive Manufacturing," Under review, 2018.

[98] O. Sigmund and S. Torquato, "Design of smart composite materials using topology optimization," Smart Materials and Structures, vol. 8, no. 3, pp. 365-379, 1999.

[99] S. Malek, J. Raney, J. A. Lewis and L. Gibson, "Lightweight 3D cellular composites inspired by balsa," Bioinspiration \& Biomimetics, vol. 12, no. 2, 2017.

[100] Z. Quan, A. Wu, M. Keefe, X. Qin and J. Yu, "Additive manufacturing of multidirectional-preforms for composites: opportunities and challenges," Material Todays, vol. 18, no. 9, 2015.

[101] K. Long, P. Yuan, S. Xu and Y. Xie, "Concurrent topological design of composite structures and materials containing multiple phases of distinct Poisson's ratios," Engineering Optimization, vol. 50, no. 4, 2018.

[102] S. Kapfer, S. Hyde, K. Mecke, C. H. Arns and G. E. Schröder-Turk, "Minimal surface scaffold designs for tissue engineering," Biomaterials, vol. 32, no. 29, pp. 6875-6882, 2011.

[103] D. Yoo, "New Paradigms in Cellular Material Design and Fabrication," International Journal of Precision Engineering and Manufacturing, vol. 16, no. 12, p. 2577-2589, 2015.

[104] D.-J. Yoo and K.-H. Kim, "An advanced multi-morphology porous scaffold design method using volumetric distance field and beta growth function," International Journal of Precision Engineering and Manufacturing, vol. 16, no. 9, p. 2021-2032, 2015.

[105] M. Afshar, A. P. Anaraki, H. Montazerian and J. Kadkhodapour, "Additive manufacturing and mechanical characterization of graded porosity scaffolds designed based on triply periodic minimal surface architectures," Journal of the Mechanical Behavior of Biomedical Materials, vol. 62, pp. 481-494, 2016.

[106] K. Wang, Y.-H. Chang, Y. Chen, C. Zhang and B. Wang, "Designable dual-material auxetic metamaterials using three-dimensional printing," Materials and Design, vol. 67, pp. 159-164, 2015.

[107] C. Wang, Y.-S. Leung and Y. Chen, "Solid modeling of polyhedral objects by Layered DepthNormal Images on the GPU," Computer-Aided Design, vol. 42, no. 6, pp. 535-544, 2010. 
[108] A. Faure, G. Michailidis, G. Parry, N. Vermaak and R. Estevez, "Design of thermoelastic multimaterial structures with graded interfaces using topology optimization," Struct Multidisc Optim, vol. 56, no. 4, pp. 823-837, 2017.

[109] G. Udupa, S. Rao and K. Gangadharan, "Functionally graded Composite materials : An overview," Procedia Materials Science, vol. 5, pp. 1291-1299, 2014.

[110] B. Zhang, P. Jaiswal, R. Rai and S. Nelaturi, "Additive Manufacturing of Functionally Graded Objects: A Review," in ASME 2016 International Design Engineering Technical Conferences and Computers and Information in Engineering Conference, Charlotte, North Carolina, USA, 2016.

[111] X. Kou and S. Tan, "Heterogeneous object modeling: A review," Computer-Aided Design, vol. 39, no. 4, pp. 284-301, 2007.

[112] A. Ramani, "Multi-Material Topology Optimization With Strength Constraints," Structural and Multidisciplinary Optimization, vol. 43, no. 5, pp. 597-615, 2011.

[113] X. Kou, G. Parks and S. Tan, "Optimal Design of Functionally Graded Materials Using a Procedural Model and Particle Swarm Optimization," Computer-Aided Design, vol. 44, no. 4, pp. 300-310, 2012.

[114] M. Bahraminasab, B. Sahari, K. Edwards, F. Farahmand, T. Hong and H. Naghibi, "Material tailoring of the femoral component in a total knee replacement to reduce the problem of aseptic loosening," Material and Design, vol. 52, pp. 441-451, 2013.

[115] D. Mahmoud and M. A. Elbestawi, "Lattice Structures and Functionally Graded Materials Applications in Additive Manufacturing of Orthopedic Implants : A Review," Journal of Manufacturing and Materials Processing, vol. 1, no. 2, 2017.

[116] A. Brunton, C. A. Arikan and P. Urban, "Pushing the Limits of 3D Color Printing: Error Diffusion with Translucent Materials," ACM Transactions on Graphic, vol. 35, no. 1, 2015.

[117] G. Gu, S. Wettermark and M. J. Buehler, "Algorithm-driven design of fracture resistant composite materials realized through additive manufacturing," Additive Manufacturing, vol. 17, pp. 47-54, 2017.

[118] G. X. Gu, C.-T. Chen and M. J. Buehler, "De novo composite design based on machine learning algorithm," Extreme Mechanics Letters, vol. 18, pp. 19-28, 2018.

[119] C. Bader, D. Kolb, J. C. Weaver and N. Oxman, "Data-Driven Material Modeling with Functional Advection for 3D Printing of Materially Heterogeeous Objects," 3D Printing and Additive Manufacturing, vol. 3, no. 2, 2016.

[120] H. Yu, S. Cross and C. Schuh, "Mesostructure optimization in multi-material additive manufacturing: a theoretical perspective," Journal of Materials Science, vol. 52, no. 8, pp. 42884298, 2017.

[121] G. Kennedy, "Large-scale Multi-material Topology Optimization for Additive Manufacturing," in 56th AIAA/ASCE/AHS/ASC Structures, Structural Dynamics, and Materials Conference, 2015.

[122] C. Wang, "Computing on rays: A parallel approach for surface mesh modeling from multi-material volumetric data," Computers in Industry, vol. 62, no. 7, pp. 660-671, 2011.

[123] J. Panchal, S. Kalidindi and D. McDowell, "Key computational modeling issues in Integrated Computational Materials Engineering," Computer-Aided Design, vol. 45, no. 1, pp. 4-25, 2013.

[124] L. Gibson, M. Ashby and B. Harley, Cellular Materials in Nature and Medicine, Cambridge, England: Cambridge University Press, 2010. 
[125] S. Zhou and Q. Li, "Design of graded two-phase microstructures for tailored elasticity gradients," Journal of Materials Science, vol. 43, p. 5157, 2008.

[126] G. Allaire, Shape Optimization by the Homogenization Method, Springer Science \& Business Media, 2002.

[127] X. Huang, A. Radman and Y. Xie, "Topological design of microstructures of cellular materials for maximum bulk or shear modulus," Computational Materials Science, vol. 50, no. 6, pp. 1861-1870, 2011.

[128] M. Ashby, "Materials and shape," Acta Metallurgica et Materialia, vol. 39, no. 6, pp. 1025-1039, 1991.

[129] M. Vlasea, Y. Shanjani, A. Bothe, R. Kandel and E. Toyserkani, "A combined additive manufacturing and micro-syringe deposition technique for realization of bio-ceramic structures with micro-scale channels," The International Journal of Advanced Manufacturing Technology, vol. 68, no. 9, pp. 2261-2269, 2013.

[130] Y. Quinsat, C. Lartigue, C. Brown and L. Hattali, "Multi-scale surface characterization in additive manufacturing using CT," in Advances on Mechanics, Design Engineering and Manufacturing, Cham, Springer, 2017, pp. 271-280.

[131] J. Martinez, J. Dumas and S. Lefebvre, "Procedural Voronoi Foams for Additive Manufacturing," ACM Trans. Graph., vol. 35, no. 4, pp. 44:1-12, 2016.

[132] J. Martinez, H. Song, J. Dumas and S. Lefebvre, "Orthotropic K-nearest Foams for Additive Manufacturing," ACM Trans. Graph., vol. 36, no. 4, pp. 121:1-12, 2017.

[133] M. P. Bendsoe, "Generating optimal topologies in structural design using a homogenization method," Computer Methods in Applied Mechanics and Engineering, vol. 71, no. 2, pp. 197-224, 1988.

[134] M. Bendsoe and O. Sigmund, Topology Optimization: Theory, Methods, and Applications, Springer Science \& Business Media, 2003.

[135] B.-T. Li, S.-N. Yan and J. Hong, "A growth-based topology optimizer for stiffness design of continuum structures under harmonic force excitation," Journal of Zhejiang University - SCIENCE A, vol. 17, no. 12, pp. 933-946, 2016.

[136] C.-Y. Wu and K.-Y. Tseng, "Topology optimization of structures using modified binary differential evolution," Structural and Multidisciplinary Optimization, vol. 42, no. 6, pp. 939-953, 2010.

[137] J. Sethian and A. Wiegmann, "Structural boundary design via level set and immersed interface methods," Journal of Computational Physics, vol. 163, no. 2, pp. 489-528, 2015.

[138] P. Vogiatzis, S. Chen and C. Zhou, "An Open Source Framework for Integrated Additive Manufacturing and Level-Set-Based Topology Optimization," J. Comput. Inf. Sci. Eng., vol. 17, no. 4, pp. 041012-10, 2017.

[139] R. Habib, T. Grossman, H. Cheong, A. Hashemi and G. Fitzmaurice, "DreamSketch: Early Stage 3D Design Explorations with Sketching and Generative Design," in ACM symposium on user interface software and technology, 2017.

[140] D. Systèmes, "Solidworks," Dassault Systèmes, 2018. [Online].

[141] Altair, "SolidThinking Inspired," Altair, 2018. [Online]. Available: https://www.solidthinking.com/inspire2018.html. 
[142] W. S. Dorn, R. E. Gomory and H. J. Greenberg, "Automatic design of optimal structures," Journal de Mecanique, vol. 3, pp. 25-52, 1964.

[143] A. Chan, The design of Michell optimum structures, Cranfield, United Kingdom: Cranfield College of Aeronautics, 1960.

[144] W. Hemp, "Studies in the Theory of Michell Structures," in International Congress of Applied Mechanics, Munich, West Germany, 1964.

[145] W. S. Hemp, Optimum Structures, Oxford, United Kingdom: Clarendon Press, 1973.

[146] W. S. Hemp and H. S. Y. Chan, Optimum design of pin-jointed frameworks, London, United Kingdom: Aeronautical Research Council Reports and Mem. No. 3632, Her Majesty's Stationery Office, 1970.

[147] M. Gilbert and A. Tyas, "Layout optimization of large-scale pin-jointed frames," Engineering Computations, vol. 20, no. 8, pp. 1044-1064, 2003.

[148] T. Sokół, "Topology optimization of large-scale trusses using ground structure approach with selective subsets of active bars," in 19th International Conference on Computer Methods in Mechanics, Warsaw, Poland, 2011.

[149] T. Sokół, "A 99 line code for discretized Michell truss optimization written in Mathematica," Structural and Multidisciplinary Optimization, vol. 43, no. 2, pp. 181-190, 2011.

[150] J. Wu, N. Aage, R. Westermann and O. Sigmund, "Infill Optimization for Additive ManufacturingApproaching Bone-Like Porous Structures," IEEE Trans. Vis. Comput. Graphics, vol. 24, pp. 11271140, 2018.

[151] T.-H. Kwok, Y. Li and Y. Chen, "A Structural Topology Design Method Based on Principal Stress Line," Computer-Aided Design, vol. 80, pp. 19-31, 2018.

[152] J. Wu, C. Wang, X. Zhang and R. Westermann, "Self-supporting rhombic infill structures for additive manufacturing," Computer-Aided Design, vol. 80, pp. 32-42, 2016.

[153] X. Song, Z. Zhang, Z. Chen and Y. Chen, "Porous Structure Fabrication Using a StereolithographyBased Sugar Foaming Method," J. Manuf. Sci. Eng, vol. 139, no. 3, pp. 031015-9, 2016.

[154] P. Zhang, J. Toman, Y. Yu, E. Biyikli, M. Kirca, M. Chmielus and A. To, "Efficient DesignOptimization of Variable-Density Hexagonal Cellular Structure by Additive Manufacturing: Theory and Validation," ASME. J. Manuf. Sci. Eng., vol. 137, no. 2, pp. 021004-8, 2015.

[155] Y. Wang, "3D Fractals From Periodic Surfaces," in ASME 2010 IDETC/CIE, Montreal, Quebec, Canada, 2010.

[156] W. Huang, Y. Wang and D. W. Rosen, "Inverse Surfacelet Transform for Image Reconstruction With Constrained-Conjugate Gradient Methods," ASME. J. Comput. Inf. Sci. Eng., vol. 14, no. 2, pp. 021005-10, 2014.

[157] D. Rosen, "Computer-Aided Design for Additive Manufacturing of Cellular Structures," ComputerAided Design and Applications, vol. 4, no. 5, pp. 585-594, 2007.

[158] A. Sá, V. Mello, K. Echavarria and D. Covill, "Adaptive voids: Primal and Dual Adaptive Cellular Structures for Additive Manufacturing," The Visual Computer, vol. 31, no. 6, pp. 799-808, 2015.

[159] C. Qi and Y. Wang, "Feature-based crystal construction in computer-aided nano-design," Comput.Aided Des., vol. 41, no. 11, pp. 792-800, 2009. 
[160] F. Xiao and X. Yin, "Geometry models of porous media based on Voronoi tessellations and their porosity-permeability relations," Computers \& Mathematics with Applications, vol. 72, no. 2, pp. 328-348, 2016.

[161] G. Dong, Y. Tang and Y. Zhao, "A Survey of Modeling of Lattice Structures Fabricated by Additive Manufacturing," ASME. J. Mech. Des., vol. 139, no. 10, pp. 100906-13, 2017.

[162] G. Dong, Y. Tang and Y. F. Zhao, "Simulation of Elastic Properties of Solid-lattice Hybrid Structures Fabricated by Additive Manufacturing," Procedia Manufacturing, vol. 10, pp. 760-770, 2017.

[163] Y. Tang, G. Dong, Q. Zhou and Y. F. Zhao, "Lattice Structure Design and Optimization With Additive Manufacturing Constraints," IEEE Transactions on Automation Science and Engineering, vol. PP, no. 99, pp. 1-17, 2017.

[164] O. Sigmund, "Tailoring materials with prescribed elastic properties.," Mechanics of Materials, vol. 20, no. 4, pp. 351-368, 1995.

[165] B. Bickel, M. Bacher, M. Otaduy, H. R. Lee, H. Pfister, M. Gross and W. Matusik, "Design and Fabrication of Materials with Desired Deformation Behavior," ACM Trans. Graph., vol. 29, pp. 63:1-63:10, 2010.

[166] C. Schumacher, B. Bickel, J. Rys, S. Marschner, C. Daraio and M. Gross, "Microstructures to control elasticity in 3D printing," ACM Trans. Graph., vol. 34, pp. 136:1-136:13, 2015.

[167] J. Panetta, Q. Zhou, L. Malomo, N. Pietroni, P. Cignoni and D. Zorin, "Elastic Textures for Additive Fabrication," ACM Trans. Graph., vol. 34, pp. 135:1-135:12, 2015.

[168] B. Zhu, M. Skouras, D. Chen and W. Matusik, "Two-Scale Topology Optimization with Microstructures," ACM Trans. Graph., vol. 36, no. 5, pp. 164:1-16, 2017.

[169] Y. Wang, "Periodic surface modeling for computer aided nano design," Comput.-Aided Des., vol. 39, no. 3, pp. 179-189, 2007.

[170] D.-J. Yoo, "Porous scaffold design using the distance field and triply periodic minimal surface models," Biomaterials, vol. 32, no. 31, pp. 7741-7754, 2011.

[171] D.-J. Yoo, "New paradigms in hierarchical porous scaffold design for tissue engineering," Materials Science and Engineering: C, vol. 33, no. 3, pp. 1759-1772, 2013.

[172] D.-J. Yoo, "Advanced projection image generation algorithm for fabrication of a tissue scaffold using volumetric distance field," Int. J. Precis. Eng. Manuf., vol. 15, no. 10, pp. 2117-2126, 2014.

[173] P. Huang, C. Wang and Y. Chen, "Algorithms for layered manufacturing in image space," in ASME Advances in Computers and Information in Engineering Research Volume 1, 2014, pp. 377-410.

[174] G. E. Schröder-Turk, W. Mickel, S. C. Kapfer, M. A. Klatt, F. M. Schaller, M. J. F. Hoffmann, N. Kleppmann, P. Armstrong, A. Inayat, D. Hug, M. Reichelsdorfer, W. Peukert, W. Schwieger and K. Mecke, "Minkowski Tensor Shape Analysis of Cellular, Granular and Porous Structures," Adv. Mater., vol. 23, no. 22-23, pp. 2535-2553, 2011.

[175] Y. Wang and D. Rosen, "Multiscale Heterogeneous Modeling with Surfacelets," Comput.-Aided Des. Appl., vol. 7, no. 5, pp. 759-776, 2010.

[176] W. Huang, Y. Wang and D. W. Rosen, "A Multiscale Materials Modeling Method With Seamless Zooming Capability Based on Surfacelets," ASME. J. Comput. Inf. Sci. Eng., vol. 17, no. 2, pp. 021007-9, 2017.

[177] T. Fast and S. R. Kalidindi, "Formulation and calibration of higher-order elastic localization relationships using the MKS approach," Acta Materialia, vol. 59, no. 11, pp. 4595-4605, 2011. 
[178] M. Baniassadi, H. Garmestani, D. Li, S. Ahzi, M. Khaleel and X. Sun, "Three-phase solid oxide fuel cell anode microstructure realization using two-point correlation functions," Acta Materialia, vol. 59, no. 1, pp. 30-43, 2011.

[179] M. Baniassadi, S. Ahzi, H. Garmestani, D. Ruch and Y. Remond, "New approximate solution for Npoint correlation functions for heterogeneous materials," Journal of the Mechanics and Physics of Solids, vol. 60, no. 1, pp. 104-119, 2012.

[180] H. Xu, D. Dikin, C. Burkhart and W. Chen, "Descriptor-based methodology for statistical characterization and 3D reconstruction of microstructural materials," Computational Materials Science, vol. 85, no. 1, pp. 206-216, 2014.

[181] A. Gupta, A. Cecen, S. Goyal, A. Singh and S. Kalidindi, "Structure-property linkages using a data science approach: Application to a non-metallic inclusion/steel composite system," Acta Materialia, vol. 91, no. 1, pp. 239-254, 2015.

[182] W. Huang, S. Didari, Y. Wang and T. Harris, "Generalized periodic surface model and its application in designing fibrous porous media," Engineering Computations, vol. 32, no. 1, pp. 7-36, 2015.

[183] M. Hansmeyer, "Digital Grotesque," 2013. [Online]. Available: http://www.michaelhansmeyer.com/digital-grotesque-I.

[184] D. Lohan, E. Dede and J. Allison, "Topology optimization for heat conduction using generative design algorithms," Struct Multidisc Optim, vol. 55, no. 3, pp. 1063-1077, 2017.

[185] J. Wang, G. Bai and X. Kong, "Single-Loop Foldable 8R Mechanisms with Multiple Modes," in New Trends in Mechanism and Machine Science, Cham, Springer, 2017, pp. 503-510.

[186] B. P. Rhoads and H.-J. Su, "The Design and Fabrication of a Deformable Origami Wheel," in International Design Engineering Technical Conferences and Computers and Information in Engineering Conference (IDETC/CIE), North Carolina, USA, 2016.

[187] S.-M. Belcastro and T. C. Hull, "Modelling the folding of paper into three dimensions using affine transformations," Linear Algebra and its Applications, vol. 348, no. 13, pp. 273-282, 2002.

[188] M. Schenk and S. D. Guest, "Origami folding: A structural engineering approach," in Origami 5: Fifth International Meeting of Origami Science, Mathematics, and Education, New York, 2011.

[189] T. Tachi, "Interactive form-finding of elastic origami," in the International Association for Shell and Spatial Structures (IASS) Symposium, Wroclaw, Poland, 2013.

[190] T. Tachi, "Freeform Rigid-Foldable Structure using Bidirectionally Flat-Foldable Planar Quadrilateral Mesh," in Advances in Architectural Geometry, Vienna, Springer, 2010, pp. 87-102.

[191] L. Zhu, T. Igarashi and J. Mitani, "Soft folding," Computer Graphics Forum, vol. 32, no. 7, pp. 167$176,2013$.

[192] J. Morgan, S. Magleby and L. Howell, "An Approach to Designing Origami-Adapted Aerospace Mechanisms," Journal of Mechanical Design, vol. 138, no. 5, p. 052301, 2016.

[193] M. Killian, S. Flory, Z. Chen, N. Mitra, A. Sheffer and H. Pottmann, "Curved folding," in ACM SIGGRAPH, Los Angeles, 2008.

[194] M. Kilian, A. Monszpart and N. Mitra, "String Actuated Curved Folded Surfaces," ACM Transactions on Graphics, vol. 36, no. 4, 2017.

[195] C. Wang, "Towards flattenable mesh surfaces," Computer-Aided Design, vol. 40, no. 1, pp. 109$122,2008$. 
[196] G. Fang, C.-D. Matte, T.-H. Kwok and C. Wang, "Geometry-based Direct Simulation for MultiMaterial Soft Robots," in IEEE International Conference on Robotics and Automation (ICRA), Brisbane, Australia, 2018.

[197] D. Rus and M. Tolley, "Design, fabrication and control of soft robots," Nature, vol. 521, pp. 467475, 2015.

[198] F. Connolly, C. Walsh and K. Bertoldi, "Automatic design of fiber-reinforced soft actuators for trajectory matching," Proceedings of the National Academy of Sciences, vol. 114, no. 1, pp. 51-56, 2017.

[199] N. H. Cohrs, A. Petrou, M. Loepfe, M. Yliruka, C. M. Schumacher, A. X. Kohll, C. T. Starck, M. Schmid Daners, M. Meboldt, V. Falk and W. J. Stark, "A Soft Total Artificial Heart-First Concept Evaluation on a Hybrid Mock Circulation," Artificial Organs, vol. 41, no. 10, pp. 948-958, 2017.

[200] M. Becker and M. Teschner, "Robust and Efficient Estimation of Elasticity Parameters using the Linear Finite Element Method," in Simulation und Visualisierung, 2007.

[201] B. Bickel, M. Bacher, M. Otaduy, W. Matusik, H. Pfister and M. Gross, "Capture and Modeling of Non-linear Heterogeneous Soft Tissue," ACM Trans. Graph., vol. 28, no. 3, pp. 89:1-89:9, 2009.

[202] H. Xu, Y. Li, Y. Chen and J. Barbic, "Interactive Material Design Using Model Reduction," ACM Trans. Graph., vol. 34, pp. 18:1-18:14, 2015.

[203] X. Zhang, X. Le, Wu, Z., E. Whiting and C. C. L. Wang, "Data-Driven Bending Elasticity Design by Shell Thickness," Computer Graphics Forum, vol. 35, no. 5, pp. 157-166, 2016.

[204] M. Skouras, B. Thomaszewski, S. Coros, B. Bickel and M. Gross, "Computational design of actuated deformable characters," ACM Transactions on Graphics (TOG), vol. 32, no. 4, pp. 82:1-10, 2013.

[205] L. Kharevych, P. Mullen, H. Owhadi and M. Desbrun, "Numerical Coarsening of Inhomogeneous Elastic Materials," ACM Trans. Graph., vol. 28, pp. 51:1-51:8, 2009.

[206] D. Chen, D. Levin, S. Sueda and W. Matusik, "Data-driven finite elements for geometry and material design," ACM Trans. Graph., vol. 34, no. 4, pp. 74:1-10, 2015.

[207] H. K. Yap, H. Y. Ng and R. C.-H. Yeow, "High-force soft printable pneumatics for soft robotic applications," Soft Robotics, vol. 3, pp. 144-158, 2016.

[208] J. H. a. H. Lipson, "Dynamic simulation of soft multimaterial 3d-printed objects," Soft Robotics, vol. 1, pp. 88-101, 2014.

[209] N. Cheney, R. MacCurdy, J. Clune and H. Lipson, "Unshackling evolution: evolving soft robots with multiple materials and a powerful generative encoding," in the 15th Annual Conference on Genetic and Evolutionary Computation, Amsterdam, The Netherlands, 2013.

[210] F. François, D. Christian, D. Hervé, A. Jérémie, G. Benjamin, M. Stéphanie, T. Hugo, C. Hadrien, B. Guillaume, P. Igor and C. Stéphane, "SOFA: A Multi-Model Framework for Interactive Physical Simulation," in Soft Tissue Biomechanical Modeling for Computer Assisted Surgery, Berlin, Heidelberg, Springer, 2012, pp. 283-321.

[211] C. Duriez, "Control of elastic soft robots based on real-time finite element method," in IEEE International Conference on Robotics and Automation, Karlsruhe, 2013.

[212] F. Largilliere, V. Verona, E. Coevoet, M. Sanz-Lopez, J. Dequidt and C. Duriez, "Real-time control of soft-robots using asynchronous finite element modeling," in IEEE International Conference on Robotics and Automation (ICRA), Seattle, WA, 2015.

[213] C. Duriez, E. Coevoet, F. Largilliere, T. Morales-Bieze, Z. Zhang, M. Sanz-Lopez, B. Carrez, D. Marchal, O. Goury and J. Dequidt, "Framework for online simulation of soft robots with 
optimizationbased inverse model," in IEEE International Conference on Simulation, Modeling, and Programming for Autonomous Robots (SIMPAR), San Francisco, CA, 2016.

[214] Z. Wang and S. Hirai, "Soft gripper dynamics using a line-segment model with an optimizationbased parameter identification method," IEEE Robotics and Automation Letters, vol. 2, pp. 19091916, 2017.

[215] A. A. Stanley and A. M. Okamura, "Deformable model-based methods for shape control of a haptic jamming surface," IEEE Transactions on Visualization and Computer Graphics, vol. 23, no. 2, pp. 1029-1041, 2016.

[216] L. Zhou, H.-J. Su, A. Marras, C.-M. Huang and C. Castro, "Projection kinematic analysis of DNA origami mechanisms based on a two-dimensional TEM image," Mechanism and Machine Theory, vol. 109, pp. 22-38, 2017.

[217] S. Tibbits, "Design to Self-Assembly," Architectural Design, vol. 82, no. 2, pp. 68-73, 2012.

[218] J. Choi, O.-C. Kwon, W. Jo, H. Lee and M.-W. Moon, "4D Printing Technology: A Review," 3D Printing and Additive Manufacturing, vol. 2, no. 4, pp. 159-167, 2015.

[219] T.-H. Kwok and Y. Chen, "GDFE: Geometry-Driven Finite Element for Four-Dimensional Printing," Journal of Manufacturing Science and Engineering, vol. 139, no. 11, p. 111006, 2017.

[220] E. MacDonald and R. Wicker, "Multiprocess 3D printing for increasing component functionality," Science, vol. 353, no. 6307, 2016.

[221] J. Ye, L. Chen, X. Li, Q. Yuan and Z. Gao, "Review of optical freeform surface representation technique and its application," Optical Engineering, vol. 56, no. 11, 2017.

[222] F. Wolfs, E. Fess, D. Johns, G. LePage and G. Matthews, "Computer aided manufacturing for complex freeform optics," Proc. SPIE 10448, Optifab, 2017.

[223] NASA/JPL-Caltech, "'Space Fabric' Links Fashion and Engineering," 2017.

[224] R. Felber, N. Rudolph and G. Nellis, "Design and Simulation of 3D printed air-cooled heat exchangers," in Solid Freeform Fabrication 2016, texas, 2016.

[225] S. Kitayama, H. Miyakawa, M. Takano and S. Aiba, "Multi-objective optimization of injection molding process parameters for short cycle time and warpage reduction using conformal cooling channel," The International Journal of Advanced Manufacturing Technology, vol. 88, no. 5-8, p. 1735-1744, 2017.

[226] Y. Wang, K.-M. Yu, C. Wang and Y. Zhang, "Automatic design of conformal cooling circuit for rapid tooling," Computer-Aided Design, vol. 43, no. 8, pp. 1001-1010, 2011.

[227] Y. Wang, K.-M. Yu and C. Wang, "Spiral and conformal cooling in plastic injection molding," Computer-Aided Design, vol. 63, pp. 1-11, 2015.

[228] X. Zhang, G. Fang, C. Dai, J. Verlinden, J. Wu, E. Whiting and C. Wang, "Thermal-Comfort Design of Personalized Casts," in UIST, Québec City, QC, Canada, 2017.

[229] L. Cheng, J. Liu and A. To, "Concurrent lattice infill with feature evolution optimization for additive manufactured heat conduction design," Structural and Multidisciplinary Optimization, pp. 1-25, 2018.

[230] J. J. Adams, E. B. Duoss, T. F. Malkowski, M. J. Motala, B. Y. Ahn, R. G. Nuzzo, J. T. Bernhard and J. A. Lewis, "Conformal Printing of Electrically Small Antennas on Three-Dimensional Surfaces," Advanced Materials, vol. 23, no. 11, pp. 1335-1440, 2011. 
[231] J. U. Lind, T. A. Busbee, A. D. Valentine, F. S. Pasqualini, H. Yuan, M. Yadid, S.-J. Park, A. Kotikian, A. P. Nesmith, P. H. Campbell, J. J. Vlassak, J. A. Lewis and K. K. Parker, "Instrumented cardiac microphysiological devices via multimaterial three-dimensional printing," Nature Materials, vol. 16, pp. 303-308, 2016.

[232] M. S. Mannoor, Z. Jiang, T. James, Y. L. Kong, K. A. Malatesta, W. O. Soboyejo, N. Verma, D. H. Gracias and M. C. McAlpine, "3D Printed Bionic Ears," Nano Letters, vol. 13, no. 6, pp. 2634-2639, 2013.

[233] A. Panesar, I. Ashcroft, D. Brackett, R. Wildman and R. Hague, "Design Framework for Multifunctional Additive Manufacturing: Placement and Routing of Three-Dimensional Printed Circuit Volumes," Additive Manufacturing, vol. 16, pp. 98-106, 2017.

[234] A. Panesar, I. Ashcroft, D. Brackett, R. Wildman and R. Hague, "Design framework for multifunctional additive manufacturing: Coupled optimization strategy for structures with embedded functional systems," Additive Manufacturing, vol. 16, pp. 98-106, 2017.

[235] "BionicANTs: Cooperative behaviour based on natural model," Festo, 2015-2016. [Online]. Available: https://www.festo.com/group/en/cms/10157.htm.

[236] K. Vidimce, A. Kaspar, Y. Wang and W. Matusik, "Foundry: Hierarchical Material Design for Multi-Material Fabrication," in Proceedings of the 29th Annual Symposium on User Interface Software and Technology, Tokyo, Japan, 2016. 